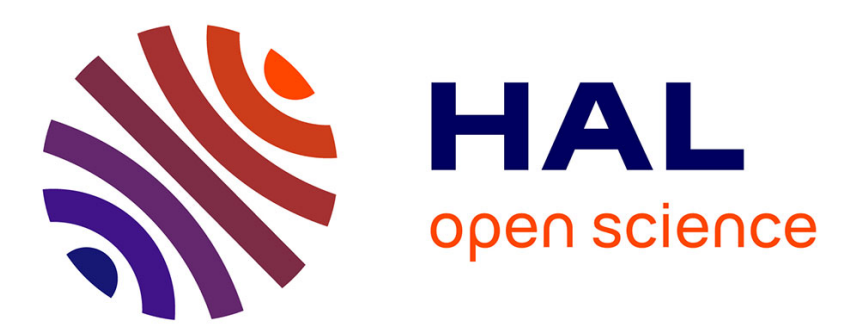

\title{
A biophysical model of brain deformation to simulate and analyze longitudinal MRIs of patients with Alzheimer's disease
}

\author{
Bishesh Khanal, Marco Lorenzi, Nicholas Ayache, Xavier Pennec
}

\section{To cite this version:}

Bishesh Khanal, Marco Lorenzi, Nicholas Ayache, Xavier Pennec. A biophysical model of brain deformation to simulate and analyze longitudinal MRIs of patients with Alzheimer's disease. NeuroImage, 2016, 134, pp.35-52. 10.1016/j.neuroimage.2016.03.061 . hal-01305755

HAL Id: hal-01305755

https://hal.inria.fr/hal-01305755

Submitted on 21 Apr 2016

HAL is a multi-disciplinary open access archive for the deposit and dissemination of scientific research documents, whether they are published or not. The documents may come from teaching and research institutions in France or abroad, or from public or private research centers.
L'archive ouverte pluridisciplinaire HAL, est destinée au dépôt et à la diffusion de documents scientifiques de niveau recherche, publiés ou non, émanant des établissements d'enseignement et de recherche français ou étrangers, des laboratoires publics ou privés. 


\title{
A Biophysical Model of Brain Deformation to Simulate and Analyse Longitudinal MRIs of Patients with Alzheimer's Disease
}

\author{
Bishesh Khanal $^{1, *}$, Marco Lorenzi $^{1,2, *}$, Nicholas Ayache ${ }^{1, *}$, Xavier Pennec $^{1, *}$
}

\begin{abstract}
We propose a framework for developing a comprehensive biophysical model that could predict and simulate realistic longitudinal MRIs of patients with Alzheimer's Disease (AD). The framework includes three major building blocks: i) Atrophy generation ii) Brain deformation iii) Realistic MRI generation. Within this framework, this paper focuses on a detailed implementation of the brain deformation block with a carefully designed biomechanics-based tissue loss model. For a given baseline brain MRI, the model yields a deformation field imposing the desired atrophy at each voxel of the brain parenchyma while allowing the CSF to expand as required to globally compensate for the locally prescribed volume loss. Our approach is inspired by biomechanical principles and involves a system of equations similar to Stokes equations in fluid mechanics but with the presence of a non-zero mass source term. We use this model to simulate longitudinal MRIs by prescribing complex patterns of atrophy. We present experiments that provide an insight into the role of different biomechanical parameters in the model. The model allows simulating images with exactly the same tissue atrophy but with different underlying deformation fields in the image. We explore the influence of different spatial distributions of atrophy on the image appearance and on the measurements of atrophy reported by various global and local atrophy estimation algorithms. We also present a pipeline that allows evaluating atrophy estimation algorithms by simulating longitudinal MRIs from large number of real subject MRIs with complex subjectspecific atrophy patterns. The proposed framework could help understand the implications of different model assumptions, regularization choices and spatial priors for the detection and measurement of brain atrophy from longitudinal brain MRIs.
\end{abstract}

Keywords: biophysical model, Alzheimer's disease, simulation of atrophy, longitudinal MRIs simulation, longitudinal modeling

\section{Introduction}

Alzheimer's Disease (AD) is one of the most common types of dementia. It is a neurodegenerative disease that progresses gradually over several years with the accumulation of

\footnotetext{
*Corresponding author

Email address: bishesh.khanal@inria.fr (Bishesh Khanal)

${ }^{1}$ INRIA Sophia Antipolis Méditerranée, Asclepios Research Project

${ }^{2}$ University College London, Translational Imaging Group, London, UK

Preprint submitted to Elsevier Journal
}

March 14, 2016 
neurofibrillary tangles (NFTs) and amyloid- $\beta$ (A- $\beta$ ) plaques [1]. These microscopic neurobiological changes are followed by the progressive neuronal damage that leads to the atrophy of the brain tissue. The atrophy or the volume changes of brain tissue is a macroscopic change that structural Magnetic Resonance Imaging (MRI) can estimate in different brain regions [2].

There is no treatment of AD so far, partly because the exact mechanisms of the disease are not well known. Nevertheless, there has been several clinical trials and disease modifying drug development efforts in the past three decades [3]. Since the external symptoms appear several years after the changes seen in imaging [2], longitudinal images can play an important role in the development of disease modifying drugs. So far, structural MRIs have primarily been used for estimating local volume changes in individual AD patients; these measurements have been used to formulate hypotheses on the temporal dynamics of AD.

An interesting alternative avenue consists in modeling the tissue loss process in order to compare (in a forward modeling setting) different hypotheses for the prediction of patientspecific time series MRIs. The ability of developing realistic individual models of brain shape changes to predict patient-specific longitudinal MRIs can have far reaching consequences. For instance, the patient specific AD trajectories predicted by the model could be useful in monitoring drug effects in $\mathrm{AD}$ patients by comparing them against the observed brain changes.

It is nevertheless very challenging to develop a comprehensive model that can predict realistic synthetic time series of MRIs following AD patient's trajectory. Modeling neurodegeneration is a complex task requiring a hierarchy of models accounting respectively i) for how and where neuronal death occurs, ii) for its effects on brain shape changes, and iii) for the subsequent brain appearance in longitudinal MRI. In Figure 1 we show a breakdown of this complex process in three major modeling blocks which represents, at a very high level, the comprehensive modeling and simulation of realistic longitudinal MRIs in AD. The first block abstracts the multi-scale models of neuronal death at the cellular level into a macroscopic map of how the atrophy spreads spatially and evolves temporally at each voxel of the brain MRI.

Knowing the patterns of local neuronal deaths and local volume loss is just one aspect of the problem; we also need to model the consequences of neuronal loss on brain shape changes. This is represented by the block Brain Deformation in Figure 1. We believe that biomechanics of brain tissue does play an important role in the way brain's shape change as a result of local volume loss, and this topic is going to be one of the main subjects of this paper.

Finally, time-series of structural MRIs capture the brain shape changes but also contain additional noise, partial volume effects and image acquisition artifacts. This is also an important aspect to consider when modeling and simulating the appearance of change in longitudinal MRIs for AD patients. This part is shown in Realistic MRI generation. Furthermore, a proper optimization framework might also be necessary to estimate the patient specific parameters of the models if we are to perform model personalization. This is represented by a feedback loop in Figure 1.

A number of atrophy simulators $[4,5,6,7,8]$ have been proposed in the literature. These 


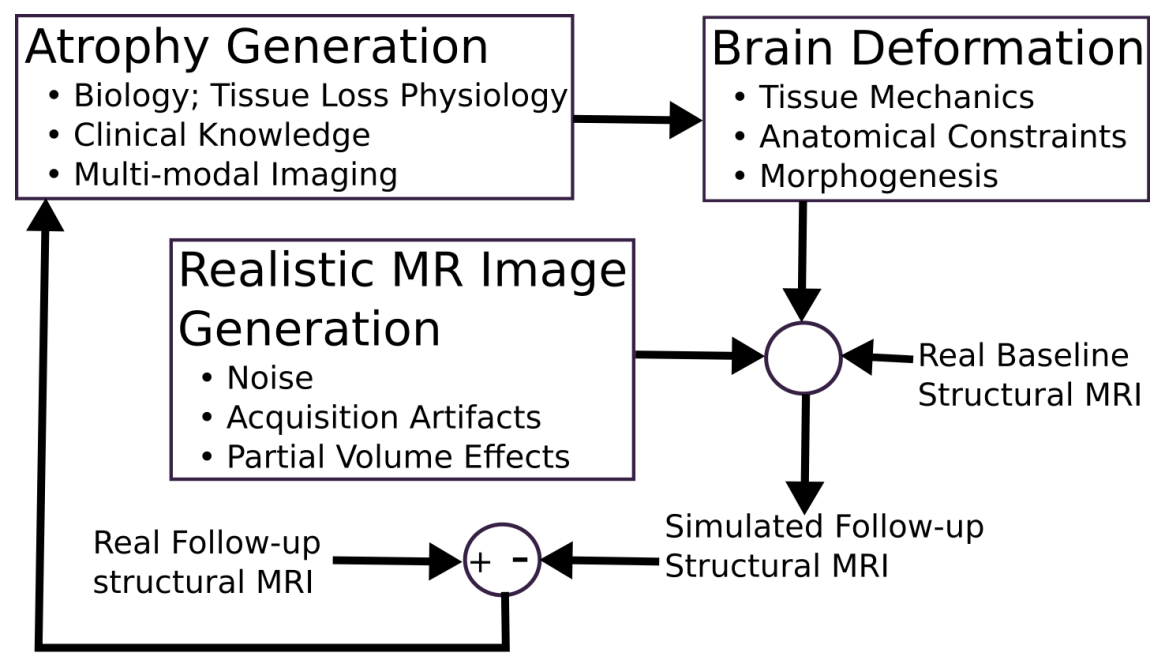

Figure 1: High level systems diagram for modeling and simulation of longitudinal MRIs in AD patients. Spatial and temporal distribution of neuronal deaths is represented in Atrophy Generation block which causes the brain shape changes represented in Brain Deformation block. This deformation along with the MRI acquisition conditions variability result intensity change in time series structural MRI of AD patients. The error in predicted follow-up from the actual observed follow-up MRI could also be used to optimize for the parameters of the developed models using a feedback system as shown above.

simulators address either just the Brain Deformation or both the Brain Deformation and Realistic MRI generation blocks in Figure 1. They propose different methods to simulate time-series images with a desired volume change. All of these simulators were developed with the objective of evaluating atrophy estimation algorithms. We can broadly distinguish two major approaches used in such simulators: Jacobian based, and biomechanical models.

In Jacobian based methods $[6,7,8]$, the desired level of atrophy is set at each voxel, and the deformation that best approximates the prescribed level of atrophy is found. Optimization of the deformation involves regularization to enforce the smoothness of the transformation and topology preservation. These simulation approaches have a number of limitations, which prevent their use and generalization in modeling oriented applications. The main issues that we identified are the following:

Plausibility and interpretation. The modeling assumptions and the regularization parameters of the energy minimization cannot be easily linked to the biophysical and mechanical process of tissue deformation. The choice of certain regularizations such as topology preservation can also have some undesirable side effects such as making it difficult to simulate the opening up of sulci.

Spatially varying tissue properties. Brain tissue and CSF are considered to respond to the volume change with the same law which is not the case in reality. Indeed, while neuronal loss in brain tissue is a gradual process, the CSF is replaced three to four times 
with the production of about $500 \mathrm{ml}$ to $600 \mathrm{ml}$ per day [9]. Jacobian-based approaches with uniform tissue properties are thus limited to explore questions such as: do different brain regions such as brain stem, cerebellum, cortex etc. respond with physical deformation in the same way to the neuronal deaths and local volume loss? Can we have parameters with a physical meaning for different brain tissue types that change the deformation we get even for exactly the same atrophy pattern? If tissues respond differently to the same amount of volume loss in brain, these models cannot accurately model the resulting shape changes and on the appearance of time-series MRIs unless the regularization is made spatially varying.

Skull invariance. In AD the brain deforms but the skull is rigid and hence the deformation model should not allow skull to move. The skull invariance is not imposed in [6]; In [8], as the authors show, imposing skull invariance results in larger error in the obtained Jacobian near the skull. Since the cortical surface lying near the skull is an important area for $\mathrm{AD}$, it is desirable not to have error in the obtained Jacobian in these areas. Finally, when only volume loss is prescribed, as seems to be the case in the evaluation experiments of $[8,10]$, it is not clear which regions of the brain expand to compensate for the volume loss since the volume within the skull must be constant when skull invariance is imposed. The spatial distribution of the resulting non-zero error in the desired vs. obtained Jacobian map is not easy to control in this case.

Biomechanical models generate tissue deformation based on biomechanical principles. As far as we know, the only model proposed so far for AD application other than the one we present here was a thermoelastic one $[4,5]$. In this thermoelastic model, one defines the volume changes in particular structures and tissues of a meshed brain by assigning different thermal coefficients. Thermoelastic model of tissue deformation is solved using Finite Element Method (FEM) to obtain a deformation field. To simulate time series of images, the deformation field interpolated from the mesh to input baseline image is used. An important limitation of this method is that it requires estimating regional thermal coefficients based on the desired volume changes which makes it difficult to prescribe complex voxel-wise atrophy patterns accurately. Although different tissue types can be differently modeled by considering tissue-specific values of thermo-elastic constants, the meaning of these parameters are difficult to link to the AD process. Furthermore, the variability of the resulting brain deformation depending on the choice of the tissue-specific parameters has not been investigated. Finally, FEM involves moving back and forth from voxels of patients MRI to reference labeled 3D mesh which creates numerical difficulties and inaccuracies in the model personalization.

In [11] we proposed a proof of concept for a new biomechanics-based tissue loss model that addresses the limitations of the previous simulators discussed above. This biophysically plausible model of brain deformation due to atrophy is constrained to fit a prescribed atrophy rate at each voxel of the parenchyma. In this work, after analyzing in detail the modeling assumptions, we provide a thorough derivation of the mathematical formulation and of the numerical implementation. There is evidence that endogenous mechanical forces at the cellular level influence brain structure and function. Although the detailed mechanisms of these interaction still deserve further investigation $[12,13]$, it is clear that they play a role at the macroscopic level which is the scale where we observe changes in the structural MRIs. 
Our model thus emphasizes, for the first time, the link between model parameters and assumptions on the real mechanobiology of neurodegeneration at a macroscopic scale. The presented experiments provide a better insight on the role of different biomechanical parameters of the model, and show that different assumptions about the atrophy process can lead to different deformations even for the same input tissue atrophy. Furthermore, we use the proposed model to study the interrelationship of various spatial atrophy patterns and how they affect the image intensity appearance. The proposed model implements the Brain Deformation block of Figure 1 and provides a mathematically solid and flexible framework to allow the future implementation of more complex modeling assumptions about neurodegeneration in the Atrophy Generation block.

Concerning the realistic MR image generation block, previous works in [14, 8, 10] provide an interesting framework for adding different kinds of intensity noise on the simulated images for the benchmarking of atrophy measurements tools. Even though this is a desirable component of a generic atrophy simulation tool, intensity noise accounts only for a small part of the variability of atrophy measurement tools. Indeed, it has been shown that the largest variability in the atrophy measurements is due to the individual variability of the brain anatomy and atrophy pattern, as well as to the wrong modeling hypothesis [10, 15]. Therefore, in this work we focus on the development of a framework that can exactly prescribe any complex pattern of atrophy in order to simulate a wide range of patient specific brain changes. To this end, in addition to the theoretical contribution on the proposed biophysical model, we provide a pipeline in order to generate a database of simulated images with complex patterns of patient specific atrophy.

In the following section we present the detailed assumptions and the development of our biophysical model of brain deformation due to atrophy. Section 3 provides the implementation details and describes how follow-up images with any desired atrophy can be simulated from any input brain MRIs. Section 4 shows some examples of such simulations. It also presents experiments that provide an insight into the role of different model parameters on the model outputs. In Section 5, we study how local and global atrophy estimation algorithms perform when a same amount of global volume changes are prescribed in two completely different ways: uniform volume changes exclusively in gray matter vs. uniform volume changes exclusively in white matter. We also present qualitative analysis of the impact of varying model parameters on the results of local atrophy estimation method for the same prescribed atrophy. Section 6 presents a framework to evaluate atrophy measurement algorithms and is illustrated by assessing the atrophy measurements in various brain structures by using representative segmentation based and registration based estimation algorithms.

\section{Biophysical Model of Brain Deformation}

The proposed model is based on a series of basic assumptions motivated by the following anatomical and biophysical notions. Human brain is enveloped by a set of membranes called meninges and the CSF that lies between the skull and the brain. Due to neurodegeneration in $\mathrm{AD}$, the brain shrinks along with some structural readjustment of the parenchyma. This 
process can be seen as a deformation of the brain parenchyma along with its mass and volume loss. The CSF volume increases to compensate the tissue volume loss while the skull remains rigid without any deformation. It is important to note that the CSF production is at a much smaller time-scale (hours) compared to the tissue atrophy (months). In the following sections we explicit the mathematical details of the tissue loss model based on these basic assumptions.

\subsection{Impact of Loss of Volume on Conservation Equation}

When modeling the deformation in AD, classical continuum mechanics formulation cannot be directly applied because conservation of mass does not hold due to the presence of atrophy. To model the brain shape changes due to neuronal deaths in $\mathrm{AD}$, it is important to take note of the observations in longitudinal brain MRIs of AD patients and also compare against other neurodegenerative diseases. In diseases like Creutzfeldt-Jakob disease, no gross brain shape changes are reported and the imaging only shows hyperintense signals on T2-weighted images [16]. However, this is not the case in AD and longitudinal MRIs show a remarkable decrease of brain volume instead [2] without any "holes". That means the tissues should restructure as the neuronal deaths increase with time. This leads us to a basic assumption in the proposed model that after the death of neurons, remodeling of the tissue occurs such that the tissue density remains constant while both the mass and volume decrease. This assumption of incompressible material but with mass loss leads us to the conservation law given by (see Appendix 1 for derivation):

$$
\nabla \cdot \mathbf{u}=-a
$$

where $\nabla \cdot \mathbf{u}$ is the divergence of a displacement field $\mathbf{u}$ associated to the deformation of the brain during a period of time $t$ and $a=a(\mathbf{x})$ is the volume loss per unit volume at position $\mathbf{x}$ during this time.

\subsection{Constrained Minimization of the Elastic Energy}

To explicitly model the neuronal loss and tissue remodeling at the microscopic level, one requires biochemical and cellular physiological knowledge in detail. These mechanisms along with the spatial and temporal evolution of the cell loss are not well known for AD. The proposed model abstracts the phenomenon that evolves during several months or years in the brain at a macroscopic scale. It is based on the assumption that atrophy creates internal stress which results in the deformation minimizing a strain energy. In other words, the brain parenchyma deforms with the prescribed atrophy by minimizing the strain energy. Using Saint Venant-Kirchoff model for an elastic material, this can be expressed as the minimization of:

$$
R(\mathbf{u}, p)=\int \mu \operatorname{tr}\left(\mathbf{E}(\mathbf{u})^{2}\right)+\frac{\lambda}{2}(\operatorname{tr}(\mathbf{E}(\mathbf{u})))^{2}-\int p(\nabla \cdot \mathbf{u}+a)
$$

where $p$ is a Lagrange multiplier, $\mu$ and $\lambda$ are Lamé constants, and $\mathbf{E}$ is Langrangian Green strain defined as: $\mathbf{E}=\frac{1}{2}\left(\nabla \mathbf{u}+\nabla \mathbf{u}^{T}+\nabla \mathbf{u}^{T} \nabla \mathbf{u}\right)$. 
By taking a sufficiently small time step $\Delta t$, this deformation could be reasonably modeled as being linear elastic. For example, for a $2 \%$ global atrophy rate per year, we have $\Delta t=$ 1 year, and the actual atrophy after one year is $a=0.02$. This linear elastic assumption is done for a small time step only because remodeling occurs to eliminate the internal stress induced by the death of neurons.

Under linear elastic assumptions, minimizing the energy in equation (2) is equivalent to solving the following system of equations.

$$
\left.\begin{array}{rl}
\mu \Delta \mathbf{u}-\nabla p & =(\mu+\lambda) \nabla a \\
\nabla \cdot \mathbf{u} & =-a
\end{array}\right\}
$$

where $\Delta \mathbf{u}$ is a component-wise Laplacian of $\mathbf{u}$. This system of equations is very similar to the Stokes flow equation in fluid dynamics [17]. The difference is in the non-zero divergence term which corresponds to the loss of matter in the tissue. The right hand side of the first equation of this system can be seen as a force term. That means the gradient of the prescribed volume loss acts as the force term, $f=(\mu+\lambda) \nabla a$, that moves the tissue for the structural remodeling. The Lagrange multiplier $p$ can be interpreted as a virtual pressure whose algebraic values can be seen as sources and sinks of matter. The second equation of this system of equations has a mass source term, $-a$.

\subsection{Modeling CSF Region}

The timescale of CSF production is hours [9], which is much smaller compared to the time scale of tissue remodeling due to atrophy. Thus the CSF should be allowed to expand as required when the brain deforms due to the prescribed atrophy. This expansion should automatically adjust and compensate for the total loss of volume prescribed in the parenchyma. For this, we release the strict constraint present in the second equation of the system of equations (3) by re-introducing the variable $p$ as: $\nabla \cdot \mathbf{u}+k p=0$, where $k$ is the compressibility with units of $\mathrm{Pa}^{-1}$. Now, the pressure in the CSF adapts to the expansion of $\mathrm{CSF}$ as required to compensate the prescribed volume loss in the parenchyma. Furthermore, since all of the CSF is considered as the fluid circulating in the brain and being constantly produced (for the timescale of months), the notion of structural readjustment due to the internal stress is not relevant. Thus the force term in system of equations (3) can also be set to zero. This leads us to the following system of equations for the CSF region:

$$
\left.\begin{array}{rl}
\mu \Delta \mathbf{u}-\nabla p=0 \\
\nabla \cdot \mathbf{u}+k p=0
\end{array}\right\}
$$

If we take the gradient on both sides of the second equation above, we have $\nabla p=$ $-(1 / k) \nabla(\nabla \cdot \mathbf{u})$. Replacing $\nabla p$ in the first equation of this system of equations, we get: $\mu \Delta \mathbf{u}+(1 / k) \nabla(\nabla \cdot \mathbf{u})=0$. This is the same equation as that of elasticity but with no external force and with $k=1 /(\mu+\lambda)$. The CSF deformation is constrained to be such that the total volume change is equal and opposite to the total prescribed tissue volume change, and the CSF-tissue interface has a continuity in $\mathbf{u}$ and $p$. How different regions of CSF 
contribute to compensate for the global volume loss depends on the choice of the value of $k$. This is further discussed in Section 4.4

The combined systems of equations for both regions are as follows:

$$
\begin{aligned}
& \text { Brain Parenchyma CSF } \\
& \left.\left.\begin{array}{rl}
\mu \Delta \mathbf{u}-\nabla p=(\mu+\lambda) \nabla a \\
\nabla \cdot \mathbf{u}=-a
\end{array}\right\} \quad \begin{array}{l}
\mu \Delta \mathbf{u}-\nabla p=0 \\
\nabla \cdot \mathbf{u}+k p=0
\end{array}\right\}
\end{aligned}
$$

Boundary conditions: As the skull is rigid and no deformation occurs in the skull, we set Dirichlet boundary conditions with zero displacement at the skull. This means that there is no flux of matter across the brain-skull interface. This interface is the skull boundary that completely encloses the CSF and brain parenchyma. In other words, setting $\mathbf{u}=0$ at the boundary $\partial \Omega$ results in $\int_{\partial \Omega} \mathbf{u} \cdot \mathbf{n} \mathrm{d} s=0$ where $\mathbf{n}$ is the vector normal to the surface $\mathrm{d} s$ of the boundary. Using divergence theorem we can re-write this surface integral as a volume integral $\int_{\Omega} \nabla \cdot \mathbf{u} \mathrm{d} \Omega=0$. Thus, with the Dirichlet boundary conditions we set, the sum of integrals of $\nabla \cdot \mathbf{u}$ in the parenchyma and the CSF should equal zero. Since we constrain the divergence in the parenchyma region with the prescribed atrophy, the system will find $\mathbf{u}$ in the CSF region such that integral of $\nabla \cdot \mathbf{u}$ over the CSF region is opposite to the integral of $\nabla \cdot \mathbf{u}$ in the parenchyma. This is how the CSF expands to compensate the volume loss prescribed in the tissue.

Material Parameters $\mu$ and $\lambda$ : The Lamé parameters are related to the material's Young's modulus $(E)$ which describes its response to mechanical stress in the following way:

$$
\lambda=\frac{\nu E}{(1+\nu)(1-2 \nu)}, \mu=\frac{E}{2(1+\nu)}
$$

where $\nu$ is a Poisson's ratio. The first Lamé parameter $\lambda$ does not have a direct physical meaning but is related to the compressibility. However, there is a strict incompressibility constraint with $\nabla \cdot \mathbf{u}=-a$ in our model. The system adapts the value of $p$ based on the chosen value of $\mu$ and the input $a$. Unlike in standard elasticity, $\lambda$ does not appear in the coefficient term of $\mathbf{u}$ and is only present in the force term $(\mu+\lambda) \nabla a$. It weights the stress exerted due to the gradient of the prescribed atrophy. Its impact on the solution is explained with experimental results in Section 4.4.

The second Lamé parameter $\mu$, also known as shear modulus or modulus of rigidity, is related to the stiffness of the material. However there is no consensus on the stiffness of the brain with widely varying estimates in the range of 0.1 to $16 \mathrm{kPa}$ [12]. Furthermore, the stiffness of the brain tissue is reported to reduce in normal aging [18] and particularly in $\mathrm{AD}$ [19]. In [20], authors estimate equivalent mechanical stress related to brain atrophy in $\mathrm{AD}$ by using strains computed from the deformation of the brain in longitudinal images and with $\mu=2.2 \mathrm{kPa}$ taken from [19]. The effect of choosing different values of $\mu$ in the proposed model is further explained in 4.4. 


\section{Implementation of the Biomechanical Tissue Loss Model}

This section describes the major implementation steps required to simulate synthetic follow-up images from an input baseline brain MRI using the proposed model of brain deformation.

\subsection{Skull Stripping and Brain Segmentation}

The model minimally requires segmentation of at least three regions of the input MRI:

i. Brain parenchyma (Gray matter/White matter region)

ii. CSF region

iii. Skull and outside region

Skull stripping separates the brain region from the skull and other outside regions. This enables us to impose Dirichlet boundary conditions of zero displacement in the skull and outside regions of the input image. Similarly, the segmentation of the brain into GM/WM and CSF enables us to numerically solve the system of equations (5). Any skull stripping algorithm, and any segmentation algorithm that can extract GM/WM and CSF can be used. The choice of algorithms/softwares used for skull stripping and segmentation in this work are detailed in later sections.

\subsection{Input Prescribed Atrophy Map}

To simulate the desired atrophy, a voxel-wise atrophy map in the GM/WM region must be provided as the input to the model. This corresponds to $a$ in equation (3). This atrophy map is thus a scalar image with desired values of volume changes in GM/WM regions, and zeros in all other regions. This image should be in the same space as the input MRI. The model can be initialized with any desired atrophy pattern, either at the regional level, or voxel-wise. Regional atrophy, such as in the whole brain, in specific brain tissues, or in regions of interests (ROIs) such as the hippocampi, can be prescribed by using any reliable anatomical segmentation tools. Otherwise, desired atrophy patterns can be generated at the voxel level by using other brain morphometry methods such as non-linear image registration (tensor based morphometry).

In the following sections we explicitly detail the brain morphometry tools chosen for each experiment. These computational methods are among the state-of-art instruments for brain morphometry, and are freely available on the web.

\subsection{Staggered Grid Discretization and Finite Difference Method}

For computing the deformation field that would warp the input image, equation (5) must be solved numerically. The computational domain for this equation is obtained from the input MRI using skull stripping and segmentation as described above. We use Finite Difference Method (FDM) with staggered grid discretization to solve the system of PDEs (5). Using staggered grid with proper placing of the pressure and velocity variable ensures stability in the solution. FDM is chosen instead of FEM to avoid brain meshing and the complexity of transporting computed variables from mesh to image at each iteration. This 
allows us to solve the system in a grid that is of the same size as the input image where the grid fits naturally to the image. This also makes it easier to obtain the partition of the computational domain into different regions directly from the skull stripping and segmentation step.

For typical brain MRIs of $1 \mathrm{~mm}^{3}$ resolution, this computational problem size becomes so large that direct solvers are impractical due to memory limitations. The system of equations (5) is similar to Stokes flow equation which is a saddle point system [21]. It needs a suitable combination of an iterative solver and a preconditioner to solve it. We use a Schur factorization to split the equations into the momentum equation and the pressure equation. Each of these equations is solved using different iterative solvers. Our implementation uses composable solvers for multiphysics with PETSc library [22] using fieldsplit preconditioner, an approach detailed in [23] with an example for Stokes flow solver with Schur complement factorization. The momentum equation is preconditioned with hypre which is an algebraic multigrid preconditioner and can be called from the PETSc interface. The implemented system is run using distributed computing in a locally available cluster.

\section{Using the Model to Explore Different Atrophy Patterns}

\subsection{Simulating Images with Any Desired Atrophy Maps}

Figure 2 shows a simulation example where a $20 \%$ hippocampal atrophy is prescribed in addition to a uniform global $2 \%$ atrophy. For this case, we used ROBEX [24] for skull stripping; FSL FAST [25] to segment the skull stripped image into GM, WM and CSF regions; and FSL FIRST [26] to segment hippocampi and amygdalae to obtain the region of enhanced atrophy. This is a simple example to illustrate that any desired atrophy can be prescribed at voxel level once the desired segmentation of the input image is obtained. Solving the model using equation (5) with the prescribed atrophy map results in a deformation field as shown in the middle of Figure 2. The deformation field is superimposed on the input baseline brain MRI. A simulated follow-up image is obtained by warping the baseline image using the computed deformation field. The difference between the real baseline and the simulated follow-up image is shown on the right of Figure 2.

\subsection{Simulating Realistic Atrophy Patterns}

We can also simulate more realistic atrophy patterns in different brain structures by taking the values reported in literature. For instance, in Figure 3 the prescribed atrophy is derived from a table in [27] that reports a mean two year atrophy of amnestic MCI patients in 35 different cortical regions. Bottom row of the figure is the computed atrophy values from the obtained deformation field. As should be the case, the divergence map is the same in the tissue while in CSF the region expands to compensate for the volume loss. We used the FreeSurfer segmentation tool [28] for the whole brain segmentation and to extract the regions of interests (ROIs) used in [27]. The whole brain segmentation with recon-all step of FreeSurfer includes skull stripping. However, FreeSurfer does not segment the sulcal CSF and only ventricular regions of CSF are segmented. Once the FreeSurfer segmentation is obtained, the sulcal CSF regions are added using the following approach: 


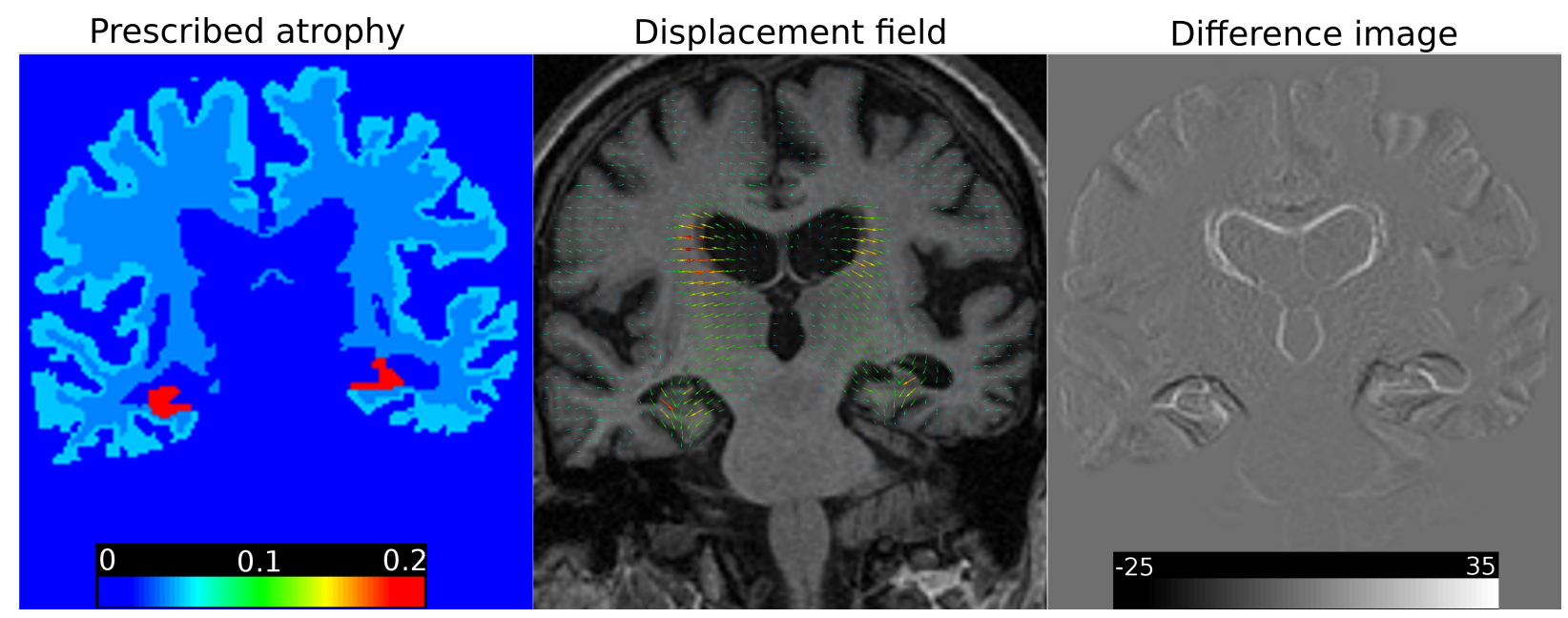

Figure 2: Atrophy in cortex, white matter and Hippocampus. No atrophy is prescribed in brain stem and other sub-cortical structures such as Thalami, Putamen etc. Left: Prescribed atrophy map with uniform atrophy of $4 \%, 5 \%$ and $20 \%$ in WM, GM and Hippocampus respectively. Middle: A coronal slice of the input MRI superimposed with the obtained displacement field. Right: Difference between the input (baseline) and the simulated (follow-up) image.

Step 1. Binarize FreeSurfer segmentation image into brain tissue vs background.

Step 2. Get a distance map of the binary image which approximates the Euclidean distance of the foreground (tissue) in number of voxels.

Step 3. In the FreeSurfer segmentation image, label as sulcal CSF the voxels that correspond to a distance less than the threshold $\beta$ in the distance map.

The chosen value of $\beta$ corresponds to the width of CSF region desired outside of the cortical surface. In the following experiments, $\beta$ was set to 2 voxels.

\subsection{Simulating Large Atrophy With Multiple Time-steps}

When the skull invariance is imposed in [8], the error in desired Jacobian vs. obtained Jacobian becomes bigger for large prescribed volume changes. With the proposed model there are no such issues and very large atrophy values can be prescribed as the model finds the deformation satisfying perfectly the prescribed atrophy constraint. In Figure 4, we see several slices a baseline and the simulated follow-up obtained by prescribing large atrophy of 90\% in Hippocampi and cortical gray matter, and 40\% atrophy in white matter. The results in the figure shown was simulated with a single time-step. Usually such large atrophies are not so common. However, we could use the model to simulate large atrophies that span over several years. In this case, the linear model with single time-step may not capture the associated deformation realistically. We can incorporate nonlinearity by solving the system of equations of the model multiple times and composing the deformation field obtained at each time-steps as explained below:

- Get the displacement field $\mathbf{u}_{0}$ by solving the model using the initial atrophy map $a_{0}$, baseline image $I_{0}$, and the segmented label image $L_{0}$ as input. 


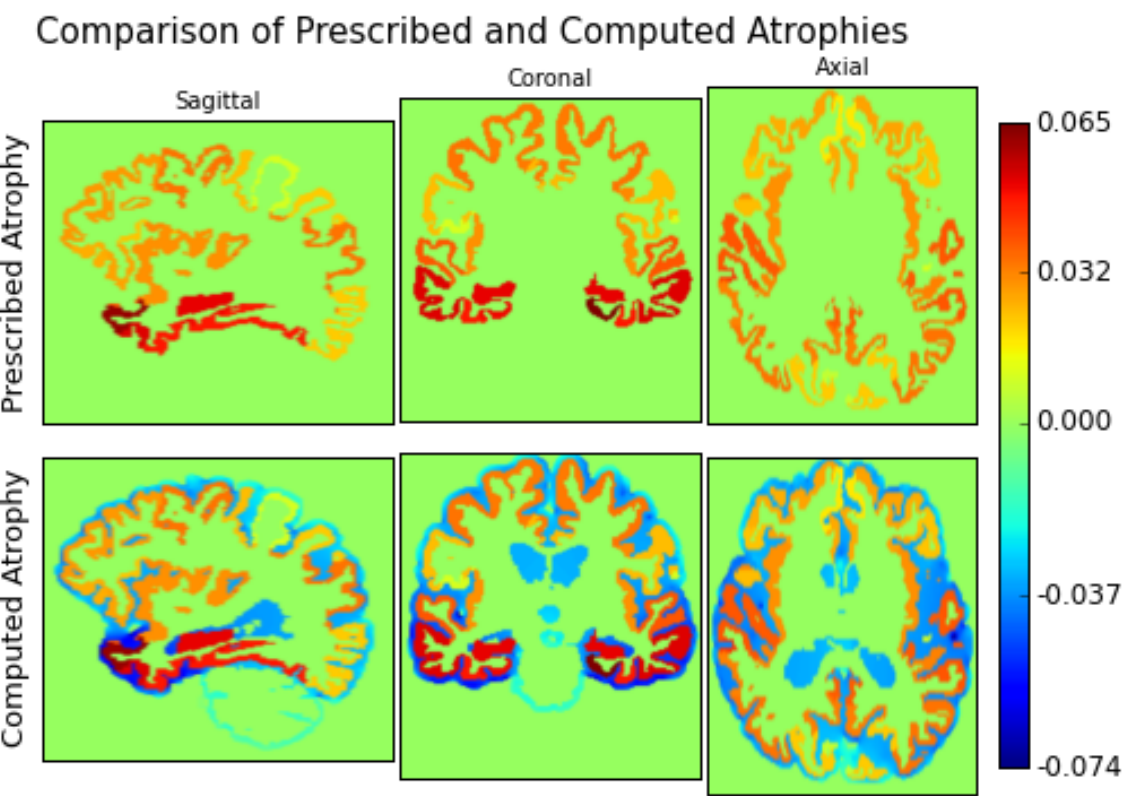

Figure 3: Prescribing uniform atrophy in 34 different cortical regions and in hippocampi. The regions are obtained from FreeSurfer whole brain segmentation. The atrophy values shown in the top row are the mean atrophy reported for amnestic MCI in [27]. The computed atrophy map is the negative of the divergence obtained from the solution of the model when solved for the prescribed atrophy. This is shown in the bottom row. The negative values of the computed atrophy map (in blue) corresponds to the expansion of CSF to compensate the volume loss prescribed in the brain tissues. We can see here that the resulting atrophy of the parenchyma is exactly the one prescribed at the voxel level while the expansion of the CSF is not uniform.

- For each time step $t=1$ to $n$ :

- Warp $a_{t-1}, I_{t-1}$ and $L_{0}$ using $\mathbf{u}_{t-1} \circ \mathbf{u}_{t-2} \ldots \circ \mathbf{u}_{0}$ to get $a_{t}, I_{t}$ and $L_{t}$ respectively.

- Solve for $\mathbf{u}_{t}$ using $a_{t}, I_{t}$ and $L_{t}$ as input.

In Figure 5, we simulate six time steps starting from an initial atrophy map measured from two MRIs of AD patient 2 years apart with the baseline age of 60 yrs. Although conceptually there is no limitation in this method to simulate very large atrophy over very long periods of time, there are nevertheless technical difficulties. If the material parameters $\mu$ is set to be discontinuous with big jump in the values of $\mu$ in different regions of the brain, the solver converges very slowly. Thus, for these cases of highly discontinuous parameters, it can become challenging to simulate large number of time steps. Similarly, when simulating multiple time steps, the atrophy map and the label image must be updated by warping them with the displacement field of the previous time step. Since the label images are required to use nearest neighbor interpolation, this can result in some of the tissue atrophy values to leak into the nearby CSF regions during the warping of the atrophy map. So, for the next step, we modify the atrophy map by redistributing uniformly all the non-zero atrophy from these CSF voxels to the nearest tissue voxels in the $3 \times 3$ neighborhood. 
We qualitatively assessed the results shown in Figure 4, by asking to an expert neurologist of the Nice Resource \& Research Memory Centre (Nice, France), to assess the plausibility of the simulated atrophy progression. According to the clinical evaluation, the pattern of morphological changes shows realistic CSF expansion at multiple scales (ventricular enlargement and sulcal widening) and cortical thinning, along with a plausible pattern of whole brain shrinkage.

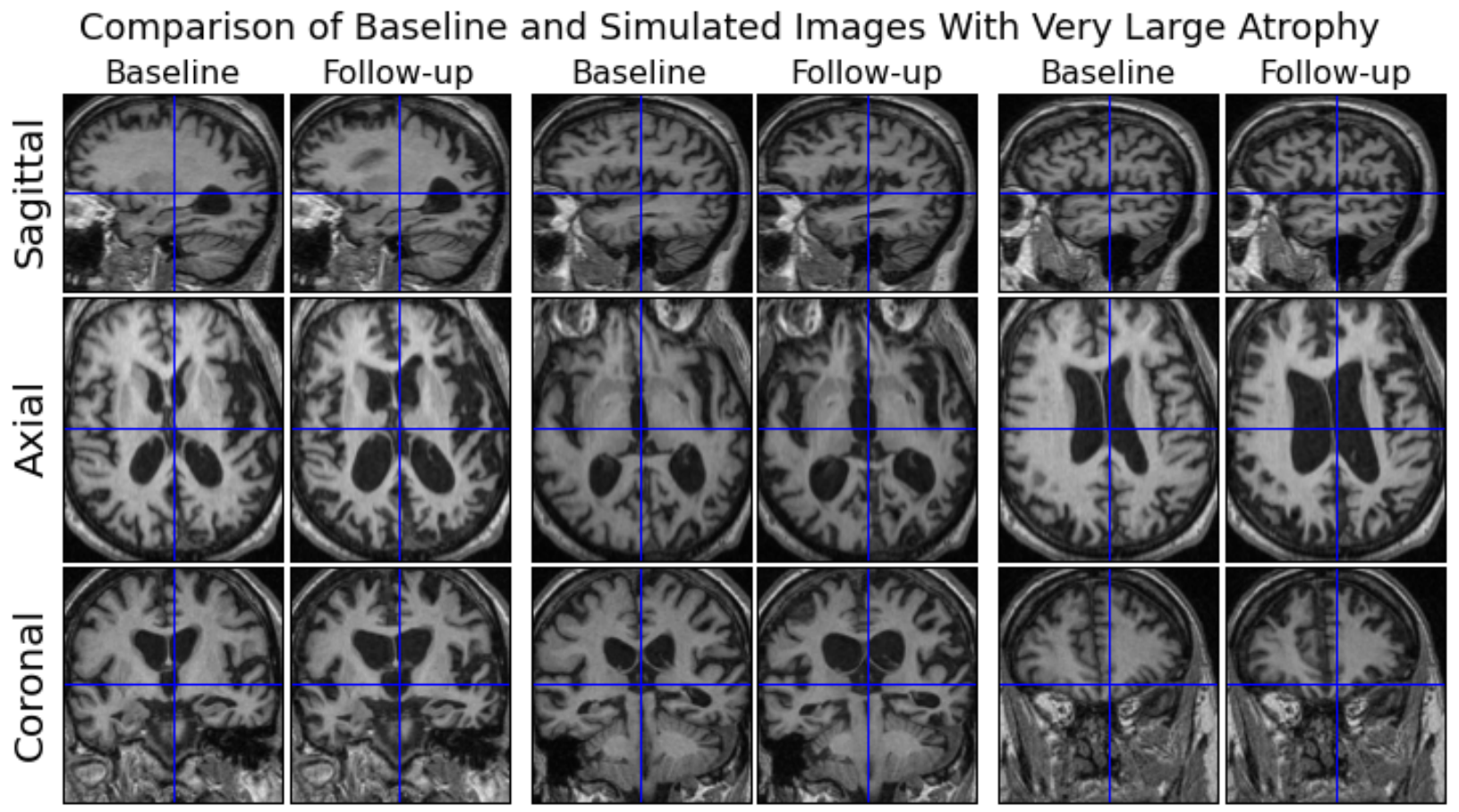

Figure 4: The figure shows several slices of a pair of real baseline and simulated follow-up MRIs with large uniform atrophy of $90 \%$ in cortical gray matter and Hippocampus, and of $40 \%$ in white matter. These large atrophies were simulated in a single time-step in this case. We can see that the model is able to simulate realistic pattern of widening and opening of sulci, the narrowing of gyri, and at the same time the cortical surface does not move unrealistically farther away from the skull.

\subsection{Role of Different Model Parameters}

Once the atrophy is prescribed and the segmentation of the input brain image is obtained, the remaining parameters that can be varied in the model are $\lambda, \mu$ and $k$. In this section we present the role of these parameters in the model. All of the numerical values presented in this work are with the following units: $\lambda, \mu, p$ in $\mathrm{kPa}, k$ in $\mathrm{kPa}^{-1}$ and $\mathbf{u}$ in $\mathrm{mm}$.

Impact of Changing $\lambda$. In standard elasticity, once the shear modulus $\mu$ is fixed, $\lambda$ is linked to the compressibility of the material as its value depends on $\mu$ and $\nu$. However, in the presented model the deformation field must satisfy the incompressibility constraint strictly and consequently $\lambda$ does not have the same usual meaning. It appears only in the equation for the brain tissue as a scaling factor to the force produced by the gradient of the prescribed 


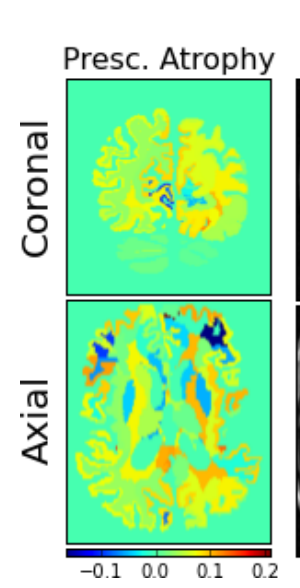

Simulating large atrophy over several years with multiple-time steps
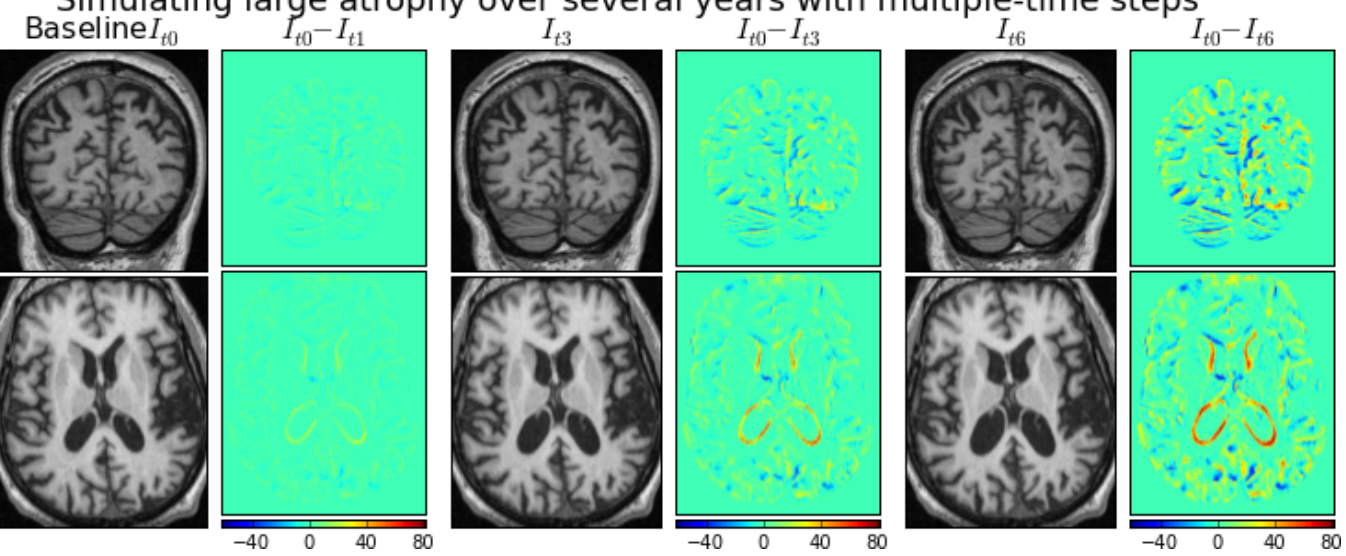

Figure 5: From left to right: Figure shows the initial prescribed atrophy, simulated follow-up images and the difference between the follow-up and baseline images for time-step 1, 3 and 6 respectively.

atrophy. Thus, the choice of $\lambda$ affects the equivalent force exerted by the gradient of atrophy and consequently the deformation field obtained from the simulation. In Figure 6, we show the results of varying $\lambda$ for the same prescribed tissue atrophy and the same values of $\mu$ and $k$. The figure shows that setting $\lambda$ too large makes the deformation field unrealistically large. Our experiments show that setting $\lambda$ to zero already provides plausible deformation field while changing $\mu$ can allow us to get different deformation fields for the same prescribed atrophy. Thus, we set it to zero in the rest of the experiments in this work.

Impact of Changing $\mu$. The shear modulus $\mu$ is the stiffness of the material and changing its values affects the deformation field obtained from the model. It appears on both the left hand side and the right hand side of Eq. 5. On the left side of Figure 7, we see the effect of varying $\mu$ in tissue and/or in CSF. When $\mu$ is same everywhere, changing it equally everywhere does not have a big effect on the deformation field but it scales up the pressure. However, when the value of $\mu$ is set differently in the tissue and the CSF, we see that the pattern of the deformation field also changes. In the last row of the figure, we see that the displacement fields are bigger near the regions where the input atrophy was non-zero. In this case the tissue is much stiffer than the CSF and for the same volume change the nearby tissue deforms to compensate for the prescribed volume changes. Different brain structures could have different material properties. However, there has not been a consensus on how these properties differ in the brain [29]. Varying the values of $\mu$ in the brain structures also produces different results for the same prescribed atrophy. For example, Figure 8 shows the difference in the simulated image between the same $\mu$ in all brain tissue vs. $\mu$ in brain stem 100 times more than other brain structures. It could be interesting to explore the impact of having different stiffness in various brain regions, or to optimize for these parameters by using multiple time-point images. This is further discussed in Section 7.

Impact of changing $k$. The compressibility coefficient $k$ is always zero in the brain tissue since we have a strict incompressible constraint. In the CSF, the choice of the value of $k$ 


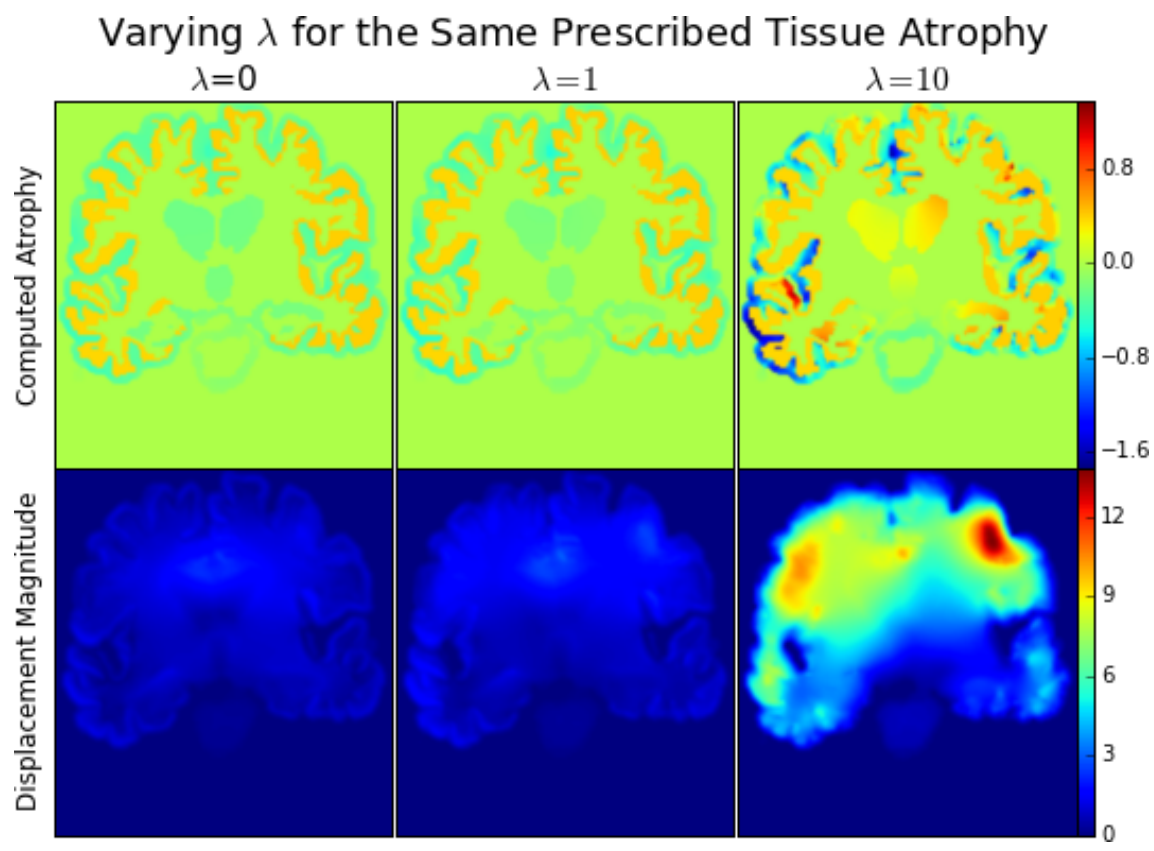

Figure 6: Setting larger values of $\lambda$ scales the force term to very high values and results in unrealistically large deformation fields.

determines its ability to expand locally. On the right side of Figure 7, we see that setting large value of $k$ allows the CSF to expand more locally in response to the nearby local tissue loss, while very small values of $k$ minimizes its expansion by distributing the same value of expansion everywhere. In a certain range of the values of $1 / k$, in the same or up to three to four orders smaller than $\mu$, it does not affect much the deformation pattern. This is the range where the variation of the redistribution of the CSF expansion in different parts of the brain is not large enough to have a big impact on the deformation field. However, if the value of $k$ is made much larger, then all of the volume loss in the tissue will be compensated by the nearby CSF regions and can even overcompensate such that the CSF in the ventricles shrink instead of expanding. This is shown in the last row of Figure 9.

\section{Investigating the Relationship Between Image Appearance and Atrophy Pat- terns}

One important question investigated in this section concerns the non-trivial effects induced by a given atrophy pattern on the appearance of the simulated images. It is important because the atrophy estimation algorithms depend on the image intensity or the appearance of the follow-up images, and the proposed model is an important instrument for studying this type of question. For this purpose we initially propose a toy example to show that different atrophy patterns can lead to very similar changes in the appearance of binary segmentations of a region of interest (ROI) (5.1), to finally provide an application in brain atrophy detection while using state-of-art brain morphometry tools (5.2). 

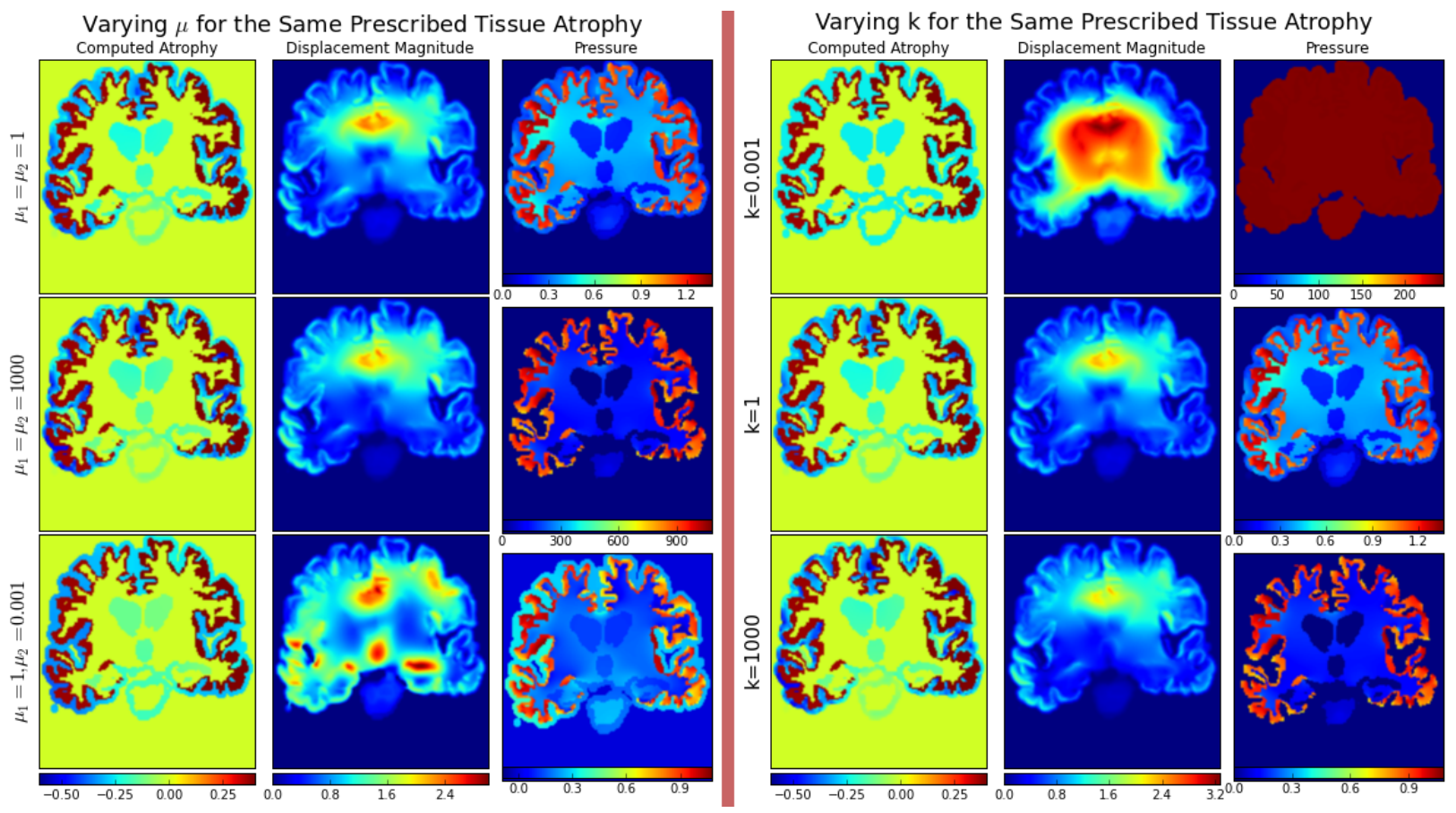

Figure 7: The prescribed atrophy is a uniform atrophy only in the cortex. Left: Effect of varying $\mu$ with $k=1 \mathrm{kPa}^{-1}$ and $\lambda=0 \mathrm{kPa} . \mu_{1}$ and $\mu_{2}$ denote the shear modulus of the brain tissue and the CSF respectively. Right: Effect of varying $k$ with $\mu_{1}=\mu_{2}=1$ and $\lambda=0$. Setting very small values for the compressibility $k$ in CSF results in its uniform expansion everywhere while allowing it to by highly compressible with large values of $k$ results in its more local expansion to compensate for the nearby tissue volume loss. The pattern of the deformation field does not change much when $\mu$ is changed in the same way in both the CSF and tissue. However, making tissue stiffer compared to the CSF makes the tissue deform differently even with the same volume loss.

\subsection{A Synthetic Example with Binary Image}

We consider a hypothetical scenario in which the segmentation of the desired brain anatomical region of interest (ROI) is provided. Our model is then applied to the anatomical region by prescribing two different atrophy patterns consisting in the same amount of global atrophy, but with different spatial distribution. In formal terms, let $a_{g}$ be a desired global atrophy of the brain and $V_{g}$ be the global brain volume. If we want $\alpha_{i}$ as the desired fraction of the total atrophy to be contributed by region $R_{i}$ with volume $V_{i}$, we have:

$$
a_{i}=\alpha_{i} a_{g} \frac{V_{g}}{V_{i}}
$$

where $a_{i}$ is the uniform atrophy needed to be prescribed in the region $R_{i}$.

The experimental scenario is tested on a cylindrical ROI, on which we generate two longitudinal deformations with different atrophy properties: the first atrophy pattern is concentrated in the medial axis, while the second one is more uniformly distributed in the volume. We note in Figure 10 that since there is not enough texture in the input image, the 


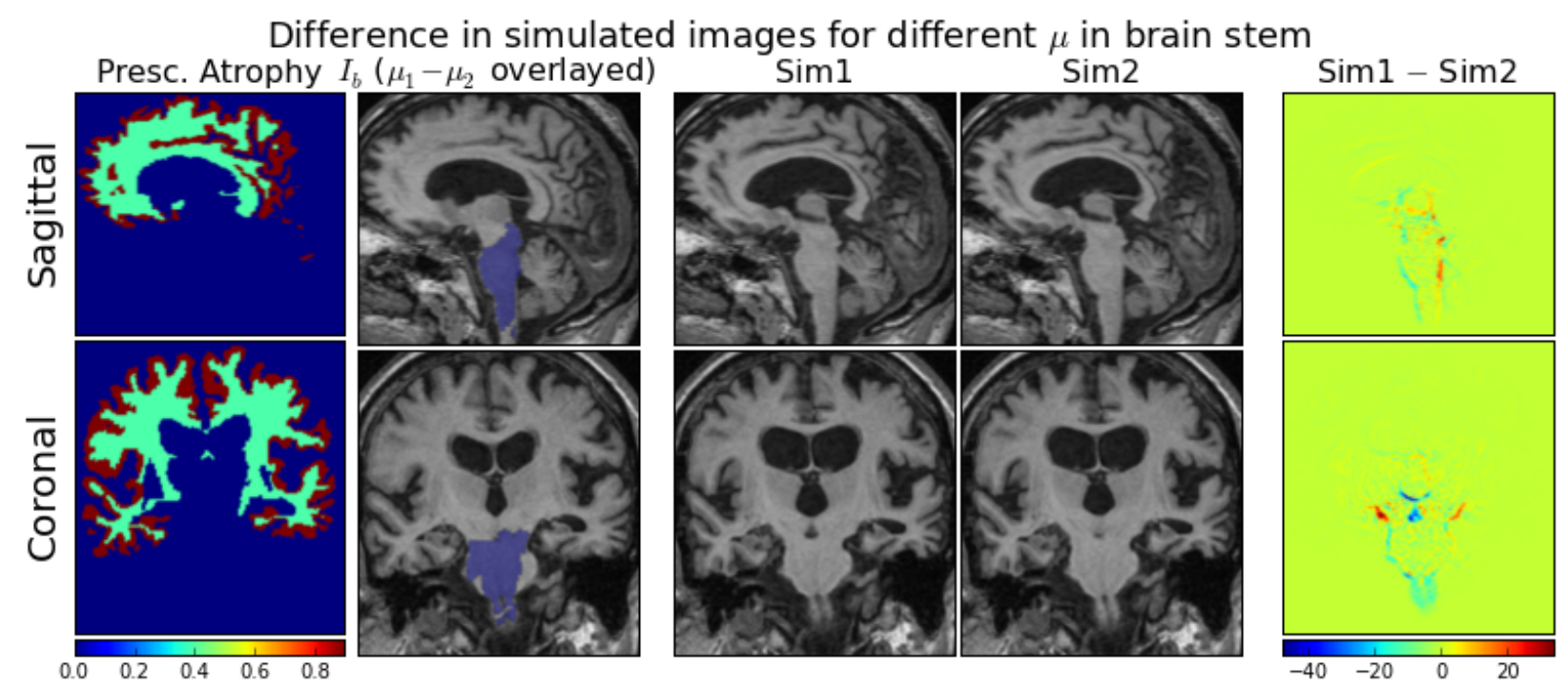

Figure 8: We see that the simulated images are different when the values of $\mu$ is changed in the brain stem to be 100 times more (difference in $\mu$ overlaid over the baseline image in second column) even when there is no atrophy prescribed in this region. The difference is more pronounced in the brain stem and nearby regions.

resulting simulated images look very similar although the underlying volume changes are very different. This is experimentally verified by non-linearly registering the simulated follow-up images to the cylindrical baseline with the LCC-logDemons algorithm [30]. Figure 11 shows that the apparent volume changes detected by the registration algorithm are very similar. Furthermore the spatial pattern of the estimated volume changes is different than both of the original input atrophy patterns. This illustrates the dependence of the registration algorithm on an implicit model (i.e. spatial regularity assumptions) when inferring the volume changes from the intensity images.

\subsection{Varying the Spatial Distribution of Atrophy in Real Brain Images}

Since the brain has a more complex shape and richer intensity information than the simple ROI considered in the previous section, it is more challenging to identify plausible atrophy based on intensity information only. It is thus of great interest to study the results of atrophy estimation algorithms when we have a same underlying global atrophy but distributed very differently in the brain. For instance, we present here atrophy estimation for two cases of simulated images having same global atrophy but different patterns: i. Only gray matter atrophy, and ii. Only white matter atrophy. We selected two representative methods of atrophy estimation: one global (gBSI [31]) and one local (LCC-logDemons[30]) for these experiments. Both of these methods are available online and are easy to use. For gBSI, there is no need to install the software locally because the input images can be uploaded to a website and the results are obtained via email [32]. 


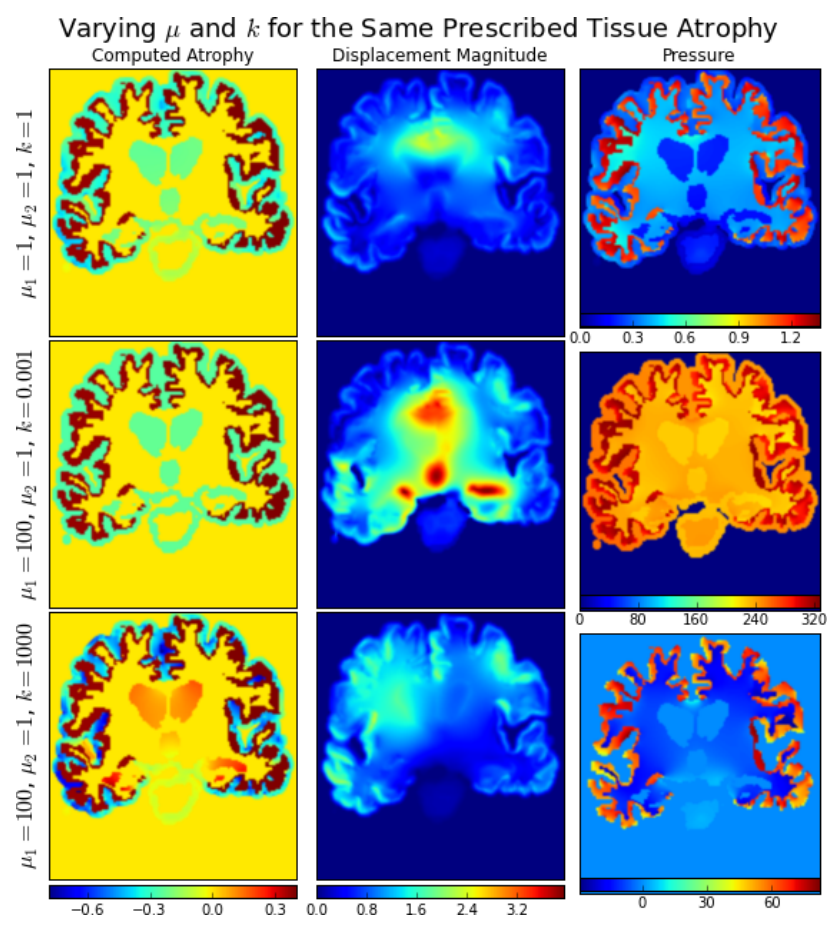

Figure 9: Impact of varying both $\mu$ and $k$. The second row shows that, just like in the previous figure, making the compressibility of the CSF $k$ much smaller compared to $\mu$ makes CSF expand uniformly everywhere. The last row shows that when $k$ is large, the CSF can expand very locally in response to the nearby tissue volume loss.

Figure 12 shows results of a very well known atrophy estimation algorithm boundary shift integral (BSI) [33] on images with same global atrophy but distributed very differently in the brain. We generated two simulated images from a single baseline MRI by prescribing global $4 \%$ atrophy either only in GM or only in WM. Figure 12 shows the brain edge movement for these two cases obtained by running the generalized BSI [31], part of NifTK software tools [32]. We see that the brain edge movement reported are similar in most areas of the brain although the underlying atrophy patterns that generated the follow-up are very different. So from a shape analysis perspective when looking at only GM-CSF interface, we are not able at all to differentiate between gray and white matter atrophy. This is quantitatively confirmed by the estimated volume change: in both cases we get the same overall volume loss in terms of total volume, and percentage change. The percentage brain volume change (PBVC) reported for the GM atrophy case was $-2.63 \%$ while for the WM case is $-2.72 \%$. The baseline (follow-up) total volume estimated are $1032 \mathrm{ml}(1011 \mathrm{ml})$ and $1032 \mathrm{ml}$ (1000 $\mathrm{ml}$ ) respectively. This shows as expected that gBSI can only estimate global brain volume change and cannot localize the atrophy to be in either gray matter or white matter.

Registration methods are usually used when one needs an estimate of local atrophy. Figure 13 shows the results of using a non-linear registration, the LCC-logDemons [30], to estimate the local atrophy pattern for the two different scenarios. In this case we notice that 


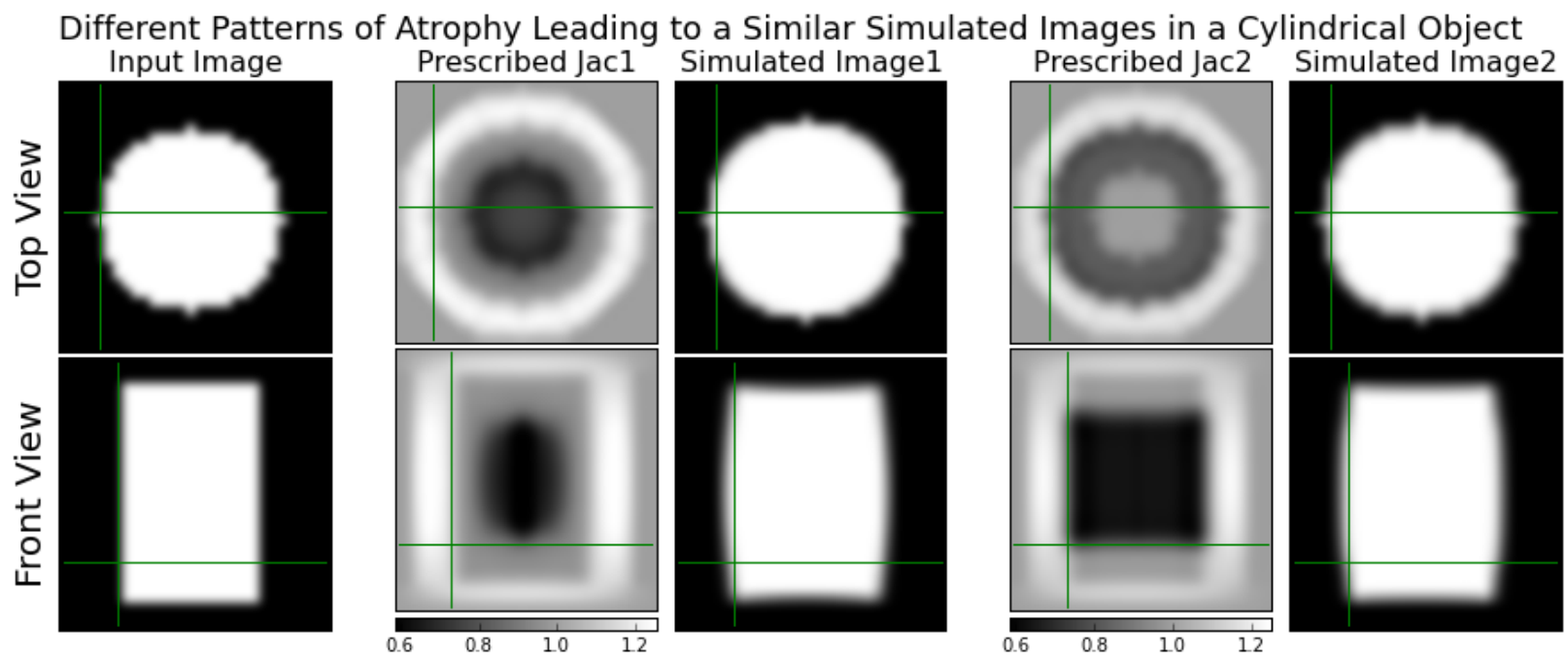

Figure 10: For a given baseline image (first column), we prescribe two different atrophy patterns (second and fourth columns). The simulated images in these cases (third and fifth columns) look very similar. The axes in the images show a reference position to aid comparison of the images. The origins of the shown axes are at the same physical position for all the images in the same row.

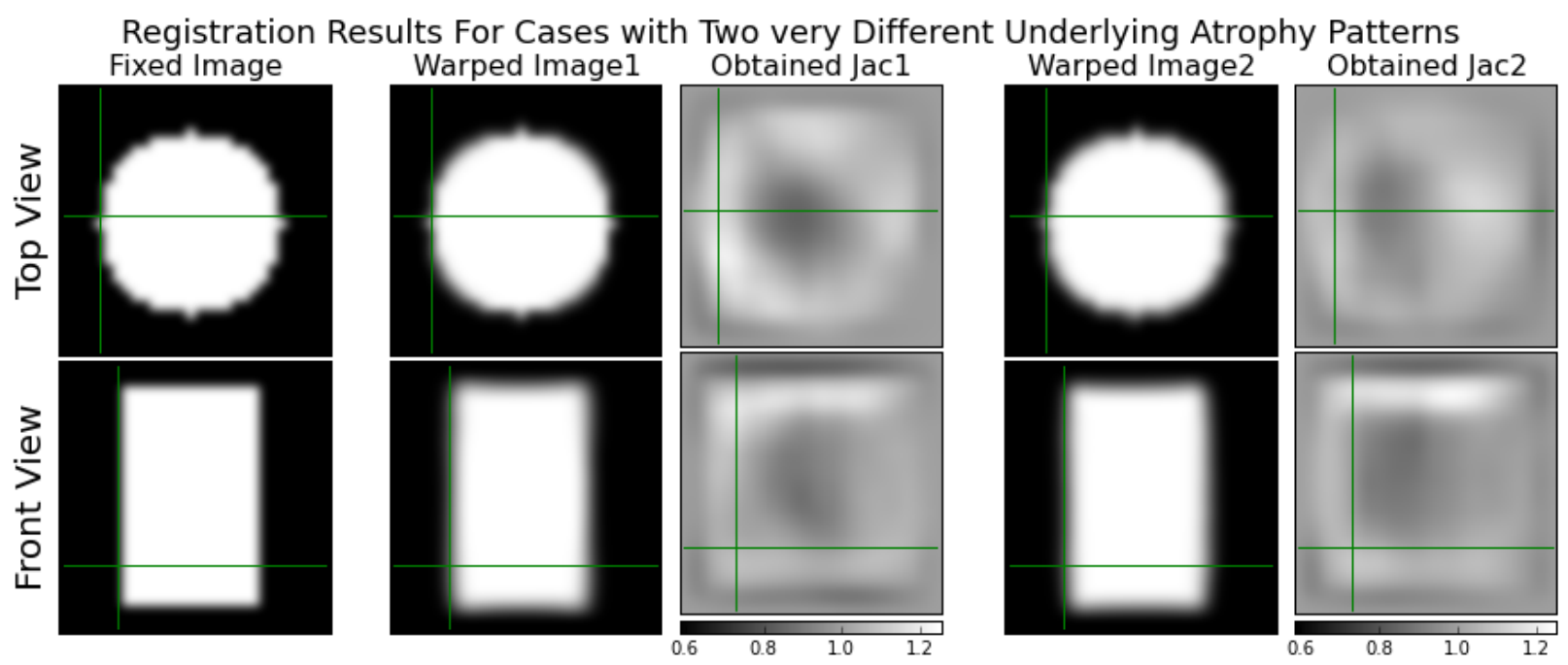

Figure 11: Registration results when using the input image of Figure 10 as a fixed image (first column) and the two simulated images of that figure as moving images (not shown here). Second and fourth columns show the results of the alignment while the third and fifth columns show the Jacobian determinants of the transformation obtained in these two cases. We see that these Jacobian determinants inferring the volume changes in the image are fairly similar. However these two moving images were created from the fixed image by prescribing very different underlying volume changes as shown in Figure 10.

the resulting atrophy patterns are different, and mostly localized in white and gray matter respectively. However, the estimated atrophy patterns are still different from the prescribed 


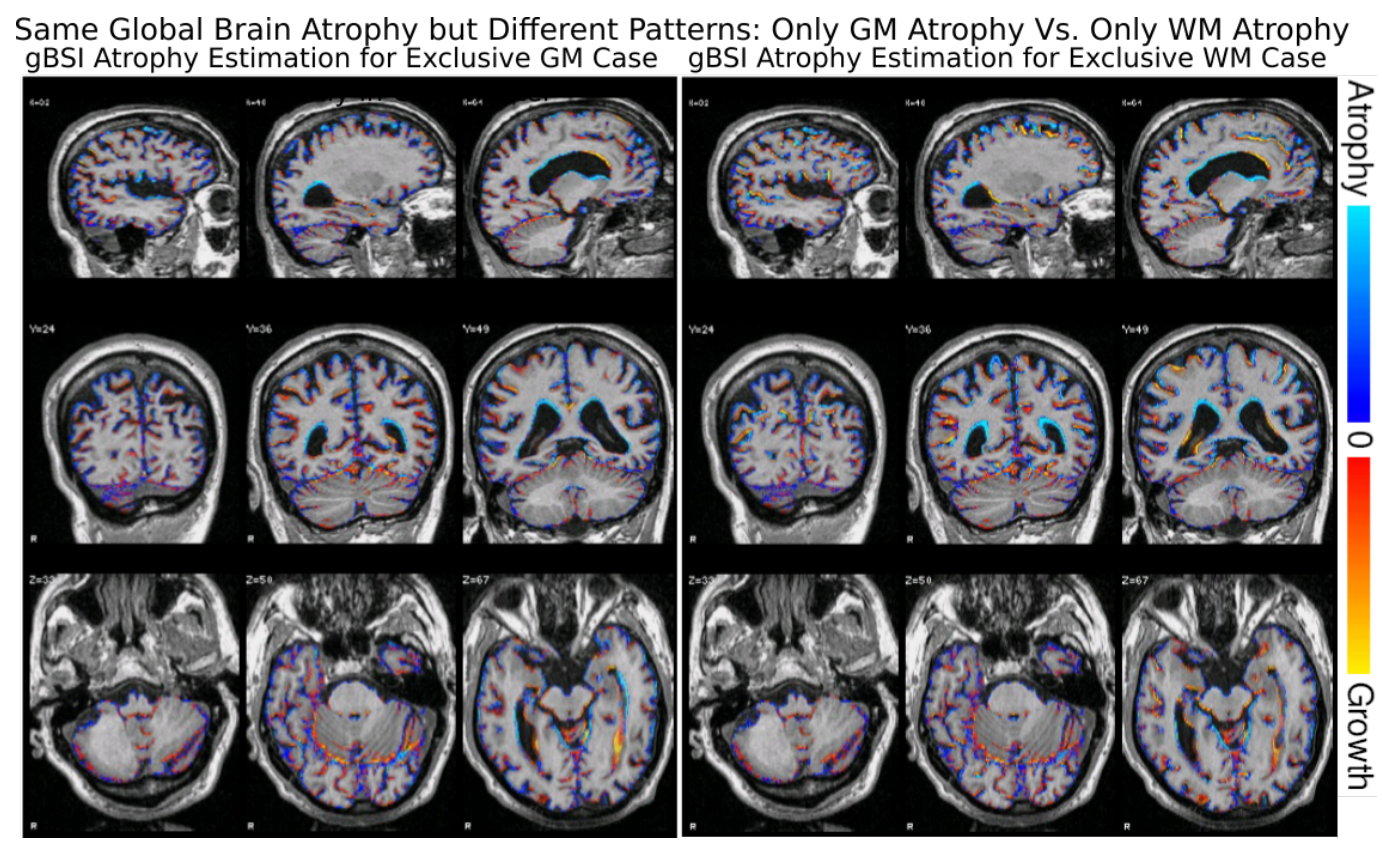

Figure 12: Brain edge movement reported by gBSI [31] for a real baseline and two different simulated follow-up images: Atrophy prescribed exclusively in Gray Matter (on left) vs atrophy prescribed exclusively in White Matter (on right). In both cases the global atrophy prescribed is $4 \%$. The underlying volume changes prescribed for these two cases are shown in the first and third columns of Figure 13. The brain edge movement and the reported atrophy are strikingly similar based on visual inspection.

ones. This is expected as the registration algorithm is unaware of the underlying model used in simulating the images. Furthermore, we also see that changing the regularization schemes changes the results of the volume changes. The two regularization schemes used were the penalization of harmonic energy and the Gaussian smoothing respectively. The harmonic energy penalization (Reg1 in the figure) results in a sharper Log-Jacobian maps while the Gaussian regularization (Reg2 in the figure) results in the more diffused atrophy. These parameters of the implicit model used in the registration algorithm are difficult to relate to the underlying model that generated the time series images.

Figure 14 shows the results of LCC-logDemons from the images simulated using exactly the same pattern of tissue atrophy but with different model parameters. The figure shows that the LCC-logDemons in general finds well the underlying volume change for both sets of parameters. However, on the left part of the figure we see that it estimates non-zero volume change in the region (shown by the axis) where no underlying volume changes were actually present. Similarly we could find for any other registration algorithm different model parameters for which the registration will produce mislocalized atrophy patterns, in particular in the areas where there are less texture such as the white matter. When estimating atrophy patterns from real observed time-series images, it is not trivial to study the relationship between the real underlying unknown deformation and the estimation provided by the algorithms such as non-linear registration. The ability to simulate different images with same 


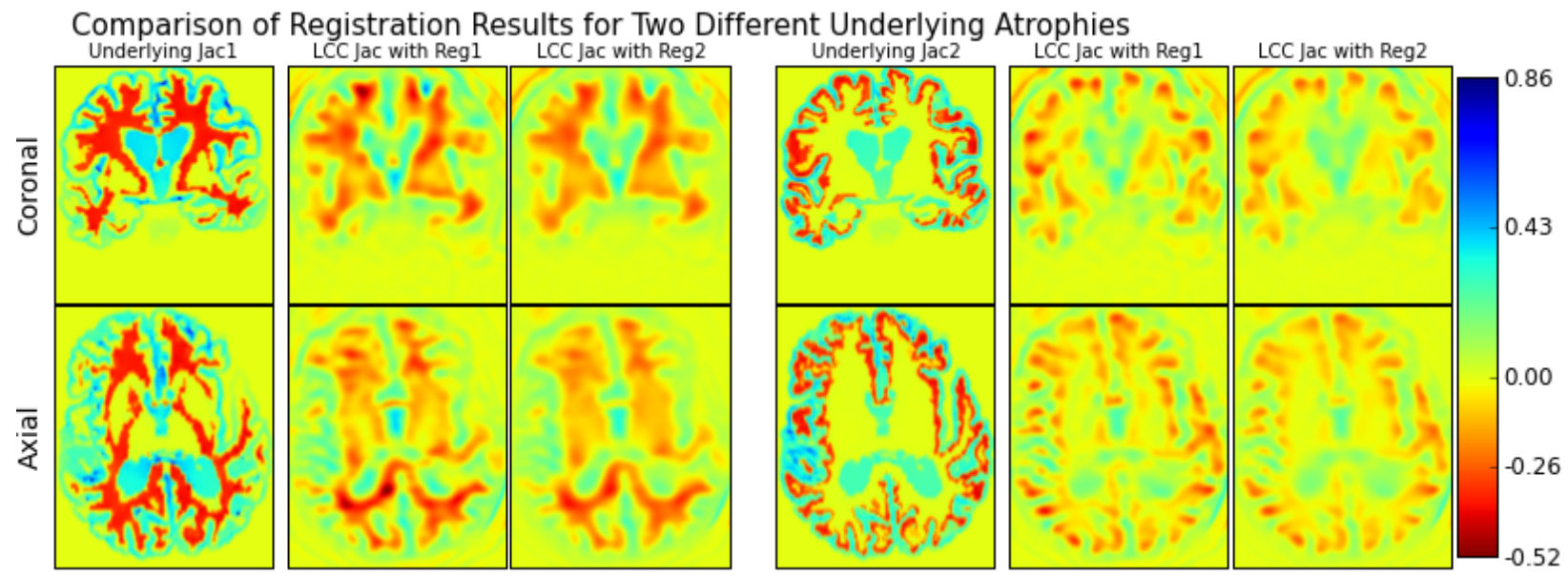

Figure 13: Estimations of volume changes using LCC-Demons registration algorithm for a real baseline and two different simulated follow-up images. The first and the third columns show the log Jacobian determinants of the transformation that was used to simulate the images. Both of them have the same global atrophy of $4 \%$ but distributed differently: exclusively in white matter (1st column), exclusively in cortical gray matter (third column). For each of these underlying volume changes we show results of the LCC-Demons using two different regularization schemes. Second and second-last columns show results when using harmonic regularization while the third and last columns show result when using traditional Gaussian smoothing regularization. The Log-Jacobians of the registration results show that they do not exactly match the actual prescribed Log-Jacobians but they more or less capture the underlying atrophy patterns. This is expected since the registration algorithm is unaware of the underlying model that generated the volume changes. When using the harmonic regularization (Reg1), the Log-Jacobian maps are sharper while using Gaussian (Reg2) results in more diffused maps.

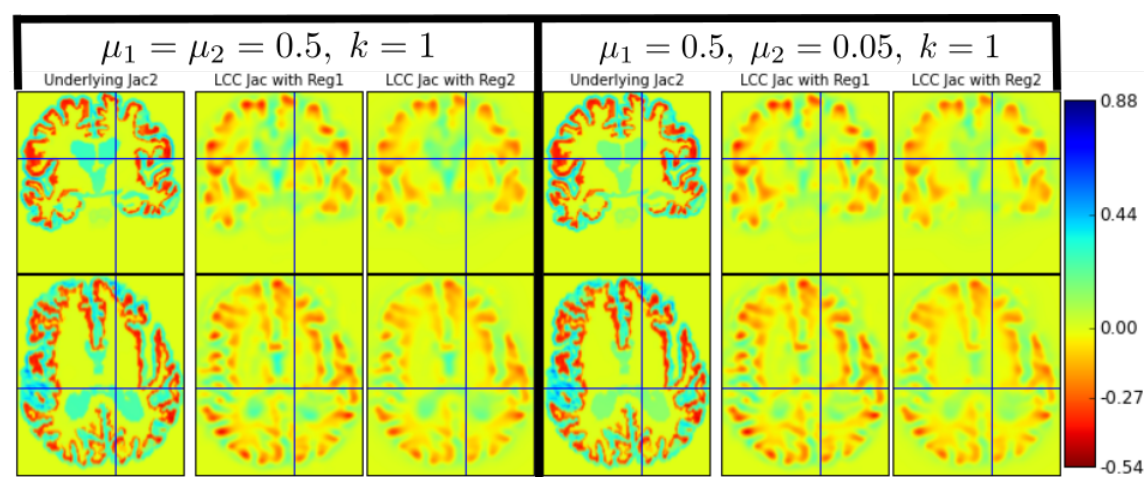

Figure 14: For the same prescribed uniform volume loss only in cortical gray matter, registration can provide different values of the measured volume changes in white matter. In the above figure we can see that in the white matter region on the right of the intersecting lines, the registration estimates volume loss differently when changing the parameters of the model without changing the underlying volume changes.

atrophy patterns but with different, biomechanically inspired, model parameters allows us to study the behavior of estimation algorithms under various assumptions. This could help 
in the future to make more informed and biologically motivated modeling choices in the development of atrophy estimation algorithms with spatially varying regularization.

\section{Simulating Complex Patterns of Patient-specific Atrophy for the Evaluation of Atrophy Measurement Algorithms}

In section 5.2 we presented a qualitative analysis of the relationship between the actual underlying deformation and the volume changes inferred by atrophy estimation algorithms. In order to provide a quantitative assessment of the simulation results, in this section we provide a framework that allows simulating patient-specific atrophy patterns in large number of patients. The framework could be used as a starting point for either calibrating the model parameters or as a framework for benchmarking atrophy estimation algorithms. The general pipeline of this framework is shown in Figure 15.

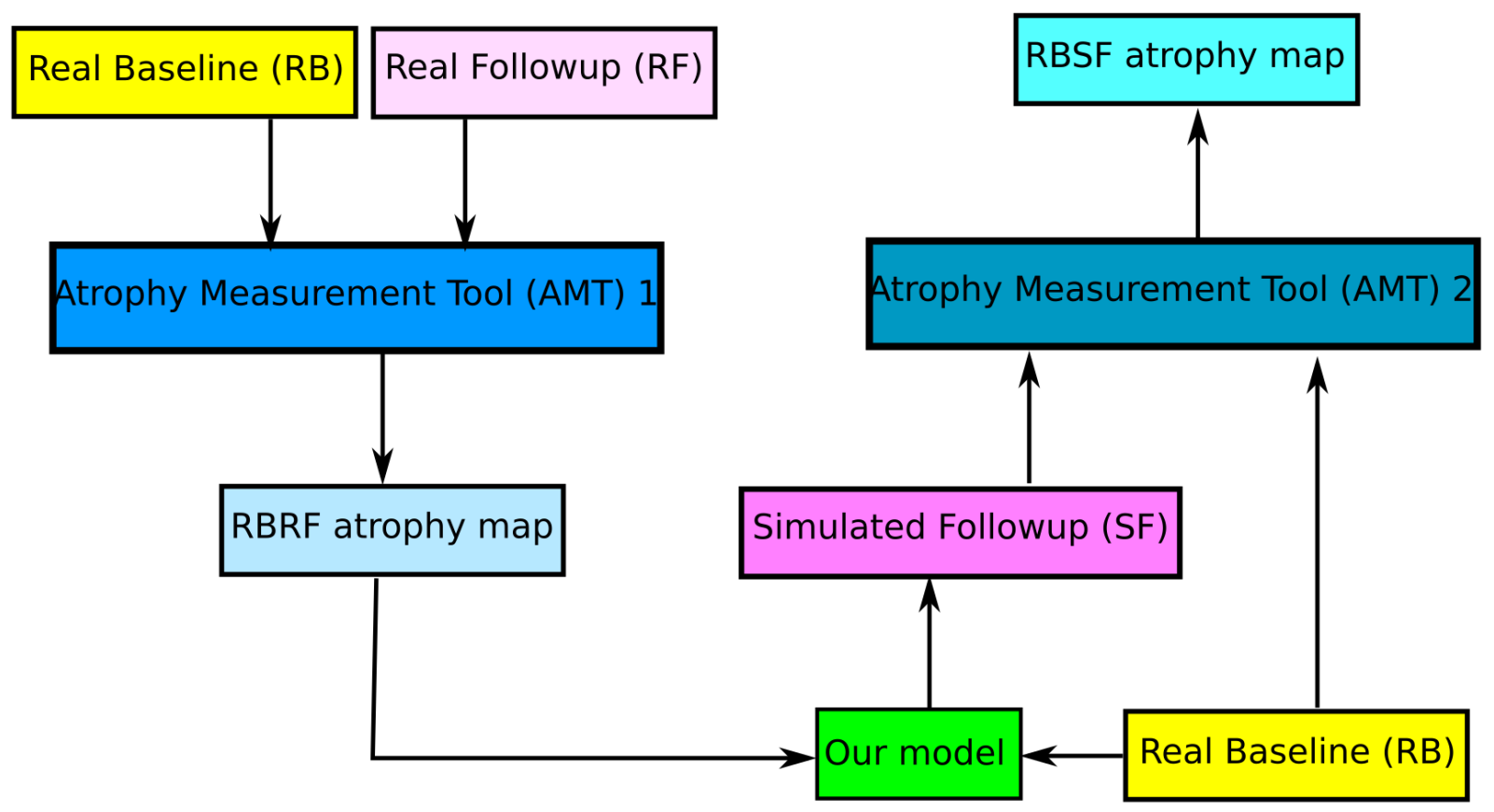

Figure 15: Pipeline illustrating the measurement of atrophy from i) RBRF: real baseline with respect to real follow-up, and ii) RBSF: real baseline with respect to simulated follow-up. The two atrophy measurement tools (AMTs) shown above as AMT1 and AMT2 can either be the same tool or different tools depending on the goals of the experiment.

The approach can be summarized in the following three steps:

1. Measure atrophy using real baseline and real follow-up image (RBRF).

2. Prescribe the measured atrophy and simulate a follow-up image. 
3. Measure atrophy again using the real baseline and simulated follow-up (RBSF).

The performance of atrophy measurement tools can thus be studied by comparing the discrepancy of the measured atrophy in the first and third step. The effect of noise and image acquisition artifacts is also an important element that could be considered while simulating the follow-up. However, this is outside the scope of the presented work, since it would require the study of reliable simulation methods to generate image artifacts such as bias field, ringing, and motion effects.

Experimental Setup. The experiments in this section uses following values for the model parameters: $\mu=1 \mathrm{kPa}, \lambda=0 \mathrm{kPa}, k=1 \mathrm{kPa}^{-1}$. The value of the shear modulus $\mu$ is in the range reported in the literature [12]. We used FreeSurfer as the atrophy measurement tool for AMT1 in Figure 15. FreeSurfer is publicly available, is widely used to study longitudinal changes in different brain regions and can segment large number of cortical, sub-cortical and white matter regions of the brain. Then we made two separate measurements of the atrophy from simulated images: i) using FreeSurfer as AMT2 in Figure 15 ii) using LCC-logDemons as AMT2.

We used T1 structural MRI of 46 Alzheimer's patients each having multiple time-point images in the range of 2 weeks to 2 years from the Miriad dataset [34]. For each of these 46 subjects following processing steps are performed:

Step 1. Create a subject specific template using all the available time-points. This uses longitudinal stream of FreeSurfer to create an unbiased subject specific template image [35].

Step 2. Get whole-brain FreeSurfer segmentation of the extremal time-point image. The first time-point corresponds to real baseline (RB) while the last time-point corresponds to real follow-up (RF) as shown in Figure 15.

Step 3. For each segmented region:

- Get the volumes reported by the segmentation in RB and RF images: $V_{0}$ and $V_{1}$.

- Compute the atrophy from the obtained volumes: $a^{\mathrm{r}}=\left(V_{0}-V_{1}\right) / V_{0}$. This results in the RBRF atrophy map of Figure 15.

Step 4. Simulate follow-up image (SF) from the RB image and the RBRF atrophy map.

Step 5. Get whole-brain segmentation of SF using the previously created subject-specific template.

Step 6. Similar to step 3, get the volume measurements in SF: $V_{1}^{\mathrm{s}}$, and atrophy estimates as $a^{\mathrm{s}}=\left(V_{0}-V_{1}^{\mathrm{s}}\right) / V_{0}$. This results in the RBSF atrophy map of Figure 15

For LCC-logDemons, we registered real baseline images with their corresponding simulated follow-up images and computed the average Jacobian determinants of the resulting deformation field in each of the ROIs provided by FreeSurfer. The volume change measure to compare against the FreeSurfer measurements is given by $J-1$, where $J$ is the average Jacobian of that ROI. We used default parameters of the LCC-logDemons for all the subjects. 


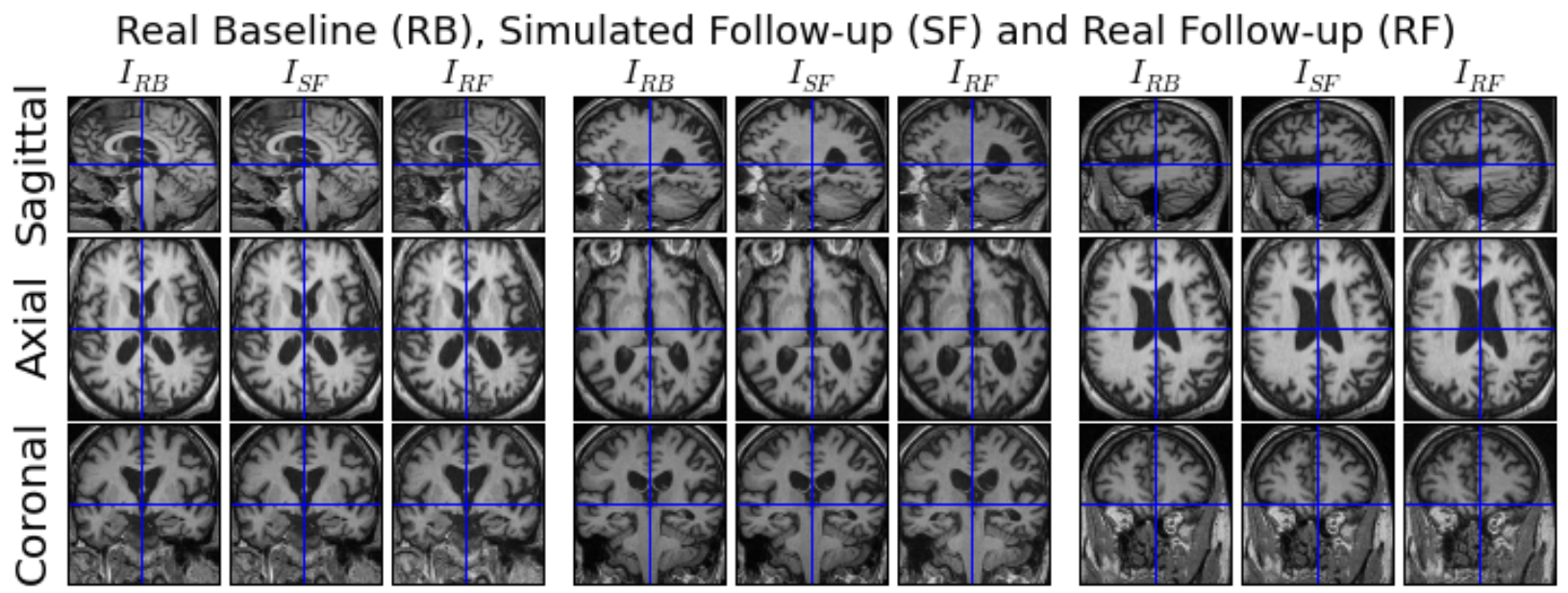

Figure 16: The figure shows nine slices of the real baseline, simulated follow-up and the real follow-up images of one of the subjects generated using the pipeline shown in Figure 15. The real follow-up image is aligned to the real baseline image using a rigid registration for visualization purpose. This allows visual comparison of the discrepancies between the simulated follow-up and the real follow-up. For this particular subject, the ventricular expansion in the simulated follow-up seems to be less than the real follow-up. It is expected that the simulated and the real follow-up do not match exactly since the atrophy prescribed to simulate the follow-up comes from an atrophy estimation algorithm which does not use the same modeling assumptions as our model.

Results. In [35], developers of the FreeSurfer longitudinal stream present test-retest reliability of the FreeSurfer segmentation by using 115 pairs of same day scans of healthy controls. The discrepancies in the volumes measured in two scans of the same patient on same day gives an idea on the variability of FreeSurfer segmentation. As a dimensionless measure of variability, they compute the absolute symmetrized percent volume change (ASPVC) of a structure with respect to the average volume:

$$
\text { ASPVC }:=100 \frac{\left|V_{2}-V_{1}\right|}{0.5\left(V_{1}+V_{2}\right)}
$$

Since we simulate follow-up images which should ideally have the same volume as their corresponding real follow-up images in the selected regions, we use the same ASPVC measure as in [35] to compare the FreeSurfer volume measurements on the real and simulated followup image pairs. Figure 17 shows mean and standard deviation of the ASPVC for two different sets of regions. The regions on the left are the twenty regions with lowest mean ASPVC while on the right are the same regions for which the results of the test-retest reliability study are available in [35]. For the regions presented in [35], we find that the mean ASPVCs from our real-simulated image pairs are in the same order as that of the test-retest real image pairs. The results show that the mean ASPVCs in most regions when using longitudinal FreeSurfer stream in our real-simulated image pairs are in between the results of cross-sectional and longitudinal stream runs presented in [35]. However, it should be noted that the result of the test-retest study is not directly comparable to our real-simulated study because the datasets 


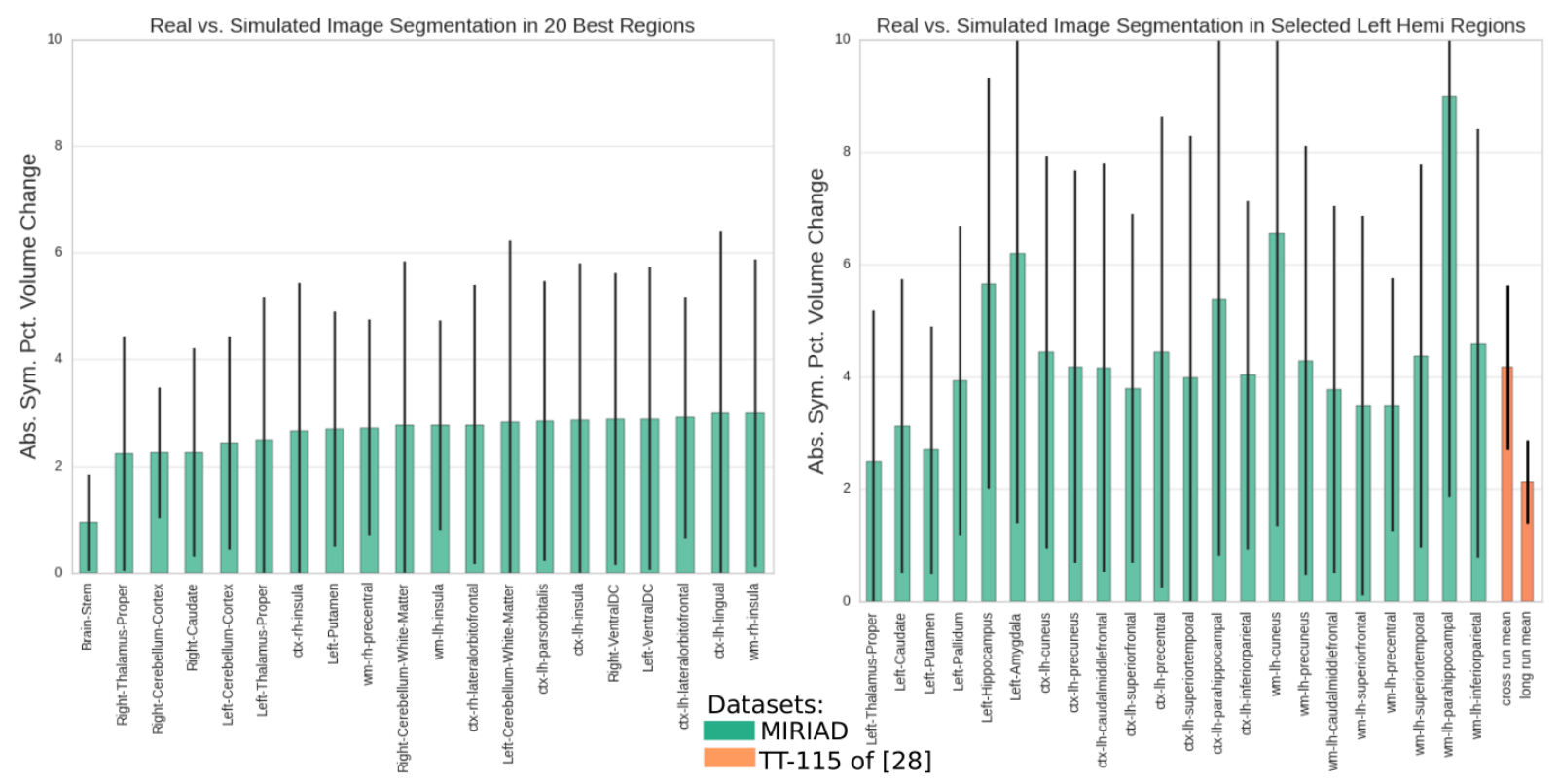

Figure 17: FreeSurfer volume measurement comparison on real follow-up vs simulated follow-up image pairs using Absolute Symmetric Percentage Volume Change (ASPVC). The top end of the bars are the mean ASPVC values while the ticks show the plus/minus standard deviation from the mean. Left: Best twenty structures for which the mean ASPVC was found to be the lowest. Right: Only the brain structures for which the mean ASPVC of FreeSurfer cross-sectional run and longitudinal run were reported in [35]. In the rightmost two bars we present the average and standard deviation of mean ASPVCs over all the available regions in Figure 7 of [35], which used a different dataset TT-115 consisting of test-retest same day repeat scan pairs of 115 healthy controls. Although not directly comparable due to the use of different datasets, we do see that the mean ASPVC computed from our real vs simulated follow-up image pairs are of the same order as that of the one presented in [35].

used are different; the dataset used in [35] is not available in the public domain. This might also have resulted in the increased variability of the computed ASPVC. For instance, a study in [36] shows that the FreeSurfer reliability on hippocampal volume measurements is non-uniform across different age groups. In particular, the study shows that the volume measurements in older age groups are not as reliable as in younger groups. Since in AD patients, the structural changes are more pronounced than in normal ageing, it is possible that the reliability will be worse in $\mathrm{AD}$ patients compared to normal ageing. In order to ascertain this effect, further test-retest study is required with several datasets of different age groups. Similarly, the amount and pattern of atrophy prescribed is different for each of the subjects. FreeSurfer volume measurements on the simulated images might be impacted differently when images are simulated with varying anatomy and atrophy patterns. Finally, the ventricles and other CSF regions are not constrained to have exactly the same volume change as the one measured from the real follow-up. These factors could also have increased the variability in the real vs. simulated repeat volume measurements.

We can also compare the distribution of atrophy estimates of the population of $\mathrm{AD}$ 


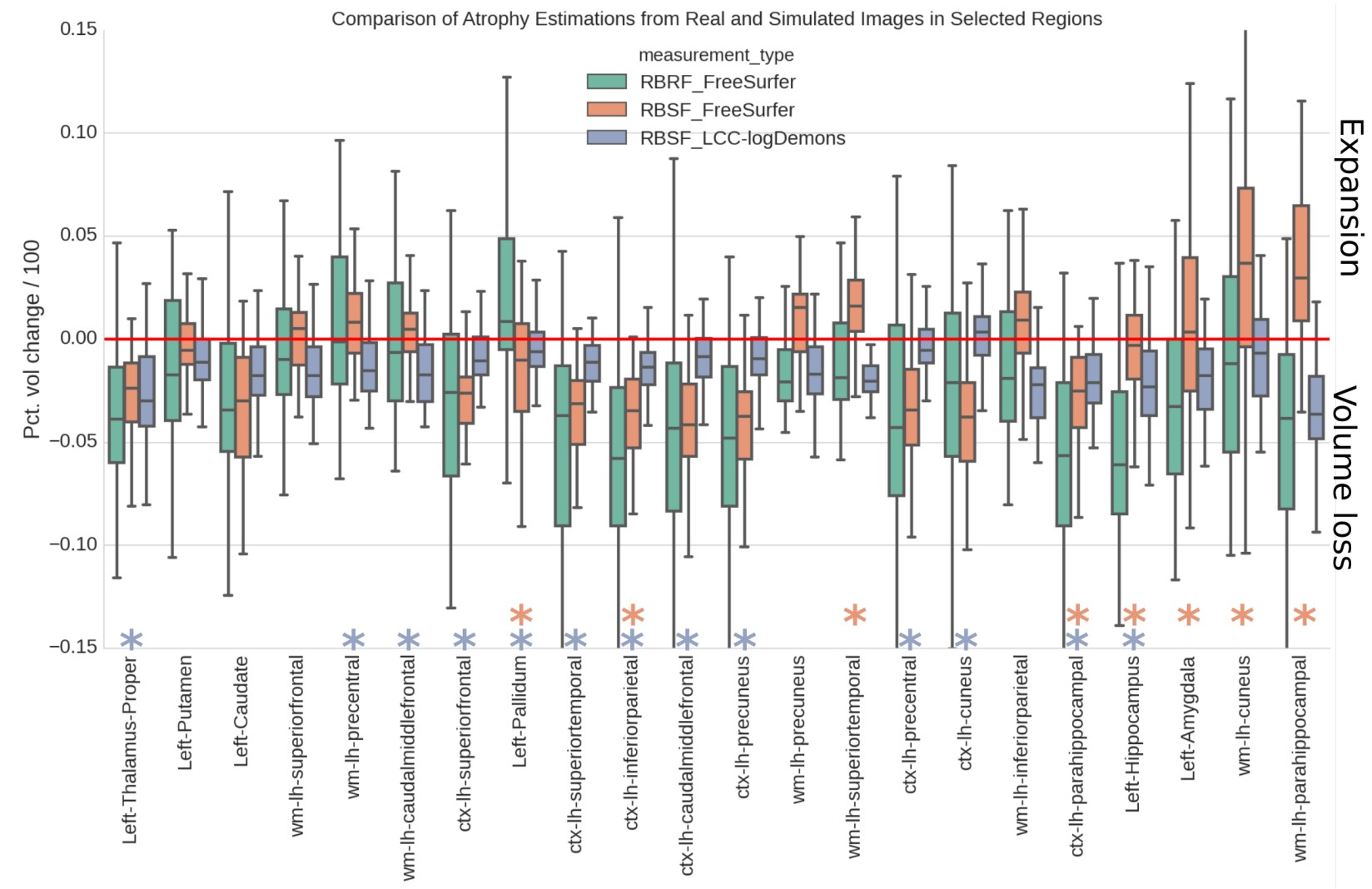

Figure 18: For each region, the box plots on the left show the estimated atrophy from the real follow-up images (RBRF atrophy map in Figure 15) using FreeSurfer, while the one on the middle and right are from the simulated follow-up images (RBSF atrophy map in Figure 15) using FreeSurfer and LCC-logDemons respectively. The brain structures shown are the regions on the left of Figure 17. We see reduced variability in the estimated atrophy of structures in the population when simulated follow-up is used. * signifies that the average atrophy of the region for the population measured from the RBSF is significantly different from the measurements obtained from $\operatorname{RBRF}(p<0.01$, two sided paired t-test). The blue * (bottom) is for LCC$\log$ Demons while the orange one (top) is for FreeSurfer. We see reduced variablity in atrophy estimation of the population when using simulated follow-up images.

patients from real follow-up images $\left(a^{r}\right)$ with that from simulated follow-ups $\left(a^{s}\right)$. The simulated follow-up images are obtained by warping the corresponding input baseline images. Thus they have two important differences from the real follow-up images: i) Image noise in the simulated images are highly correlated to the noise in their corresponding baseline images, while the noise in the real follow-up images are independent from the noise in the baseline images. ii) The simulated images are obtained by resampling the baseline image and hence are smoother than the real follow-up images. Both of these factors can be expected to reduce the variability of the measured atrophy in the population when using simulated follow-up images instead of real follow-up images.

Figures 18 and 19 show the atrophy estimates in the MIRIAD dataset using real followups and using the simulated follow-ups. We see that, as expected, variability in atrophy 
estimates in the population is reduced remarkably when using the simulated images. Most regions show a trend of underestimation of atrophy but again there are some regions such as the white matter of cerebellum in Figure 19, and Pallidum and cortical Cuneus in Figure 18 where we observe an overestimation of the underlying atrophy with FreeSurfer. The observed variability in atrophy measures of different regions in the proposed controlled scenario could also be related to the non-uniform reliability of the atrophy estimation algorithms in different brain regions. For instance, non-uniform reliability of FreeSurfer segmentation for different structures can also be seen in the result of test-retest repeat scan experiments presented in [35].

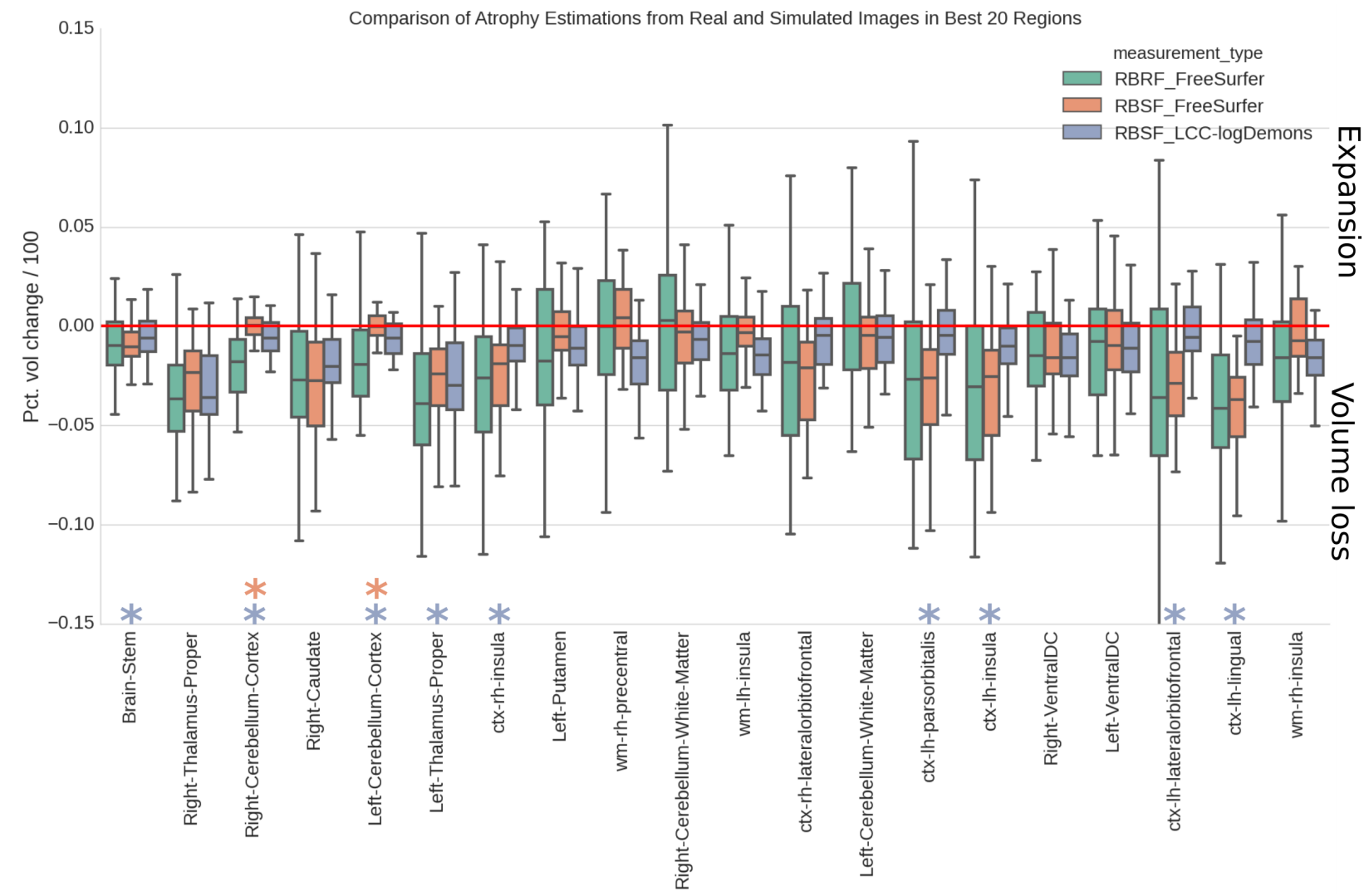

Figure 19: Same as Figure 18 but the brain structures shown are the regions on the right of Figure 17 (20 regions with least ASPVC). We again see that the atrophy estimated in the population from the simulated images has reduced variability compared to the one from real images. The atrophy estimations in real and simulated images are significantly different only for two regions when using FreeSurfer, but for nine regions when using LCC-logDemons

\section{Discussion}

In this paper we focused on the development of a brain deformation model from a known map of local volume changes, and on the study of the impact of local tissue loss on brain shape changes. 
In section 4.4 we showed that by changing the model parameters we obtain different deformation fields even for the same input atrophy. Setting different values of shear modulus in various brain regions can produce different deformations as seen from an example in Figure 8 where we set higher value of shear modulus in brain stem. It will be interesting in the future to study if one can optimise the values of $\mu$ to obtain even more realistic morphological changes in very specific structures as expected by the neurologists, such as the rotation of temporal poles in the coronal view or the movement of inferior part of the temporal lobe farther away from the cerebellar tentorium. If this set of stiffness parameters corroborates well with the values reported in the literature, it could provide better understanding of the response of various brain structures to the local volume loss.

The framework presented in 15 could also be used to calibrate the model parameters for a particular AMT. In Figures 18 and 19 we see that LCC-logDemons does not perform the same way as FreeSurfer. The result could be explained by noticing that the prescribed atrophy used in simulating images are piecewise continuous since it was computed from the segmented volumes from FreeSurfer but the model used by LCC-logDemons promotes smoothly varying Jacobian determinants. These results were obtained by using only one set of model parameters; by defining a suitable parameters optimization strategy we could obtain a minimum discrepancy in the atrophy measurements from the real and simulated follow-up images. Once the model is calibrated to a particular AMT this way, the pipeline in 15 can be used to study the relative bias of other AMTs.

One interesting future work concerns the optimization of the atrophy parameters to best explain the observed longitudinal images. This is akin to registration methods where one finds a best explanation of the observed changes based on some implicit models of regularization. In this case we have an explicit biophysical model of deformation with the advantages described earlier in the paper. The pipeline shown in Figure 15 can be useful in such optimization framework too. The RBRF atrophy map could be used as an initialization of the atrophy parameters for the optimization that minimizes the error between RBRF and RBSF. There are two major challenges that need to be taken care of in this regard. The first one concerns the very large number of parameters: since the atrophy is prescribed in every voxel, the number of parameters equals the number of voxels in the image, or the number of considered regions of interest. The second issue is the computational time for solving the model. It requires from around thirty minutes to few hours in a locally available cluster computing resource using 80 cores (depending on the choice of model parameters, and the load in the cluster from other users) to solve the model for brain MRIs of around $1 \mathrm{~mm}$ resolution. This means special efforts will be required to develop the optimization framework in a computationally feasible manner for the given image size and number of selected atrophy parameters.

The experiments and results presented in section 6 are for the illustrative purpose of using the framework in different scenarios. These experiments are not a full fledged benchmarking of the atrophy estimation algorithms as it is not the primary focus of this paper. Extensive analyses are required for proper evaluation and to find out the discrepancies in the atrophy measurement from the real and simulated follow-up images. For instance, atrophy in each ROIs can be distributed differently such as being concentrated towards the middle of the 
ROIs or in the boundaries. This could impact the contrast in the edges of each of these ROIs and consequently affect the atrophy estimations. Similarly, simulated follow-up images were obtained by resampling the intensity of the baseline images and are in the same physical space as their corresponding baseline images. This way of simulating images could possibly have failed to reproduce some of the information available in the real follow-up images (such as high intensity contrast) that are used by the atrophy estimation algorithms. Answering such questions with conclusive evidence requires additional sets of experiments studying the origins of bias and variability, and the impact of different ways of simulating ground truth images on the estimation algorithms. These experiments should also be carried out on a number of additional atrophy estimation algorithms than the ones we presented in this paper and it will be the focus of one of our future studies.

In [10], the authors create a database of simulated images from 18 MRIs by simulating uniform hippocampal atrophies in the range of $1-14 \%$ with step size of $1 \%$. For each pair of atrophy value and patient image, a number of simulated images are created by degrading the simulated atrophies with independent Gaussian noise. The authors estimated bias in the atrophy estimation of hippocampus using the simulated database and also developed a framework to provide confidence intervals of the atrophy estimation. The nature and magnitude of bias computed were based on the database containing simulations of the images with atrophy only in a single region. The framework presented by the author and the related database can be enriched by using the model we proposed in this work. We can simulate more images for the same prescribed atrophies in a particular region by varying the model parameters, and by varying the atrophy patterns in other brain regions. This allows studying the nature of bias due to the variation in the underlying model of deformations and in the presence of complex atrophy patterns in multiple brain regions. In [27], the authors use all the cortical regions segmented from FreeSurfer to identify patterns of coordinated atrophy distributed in Gray Matter of aMCI patients. The objective of that study was to explore the distributed network account in AD by studying how different groups of cortical regions are correlated to best explain the longitudinal change. Estimating bias in the measurements from atrophy estimation algorithms in the presence of atrophy in large number of structures simultaneously can be useful in assessing results of such studies too.

Towards an Integrative Multimodal Model. The anisotropic nature of the brain parenchyma due to fibers could have an impact on the way it deforms due to atrophy. Since not much is known about this, the proposed model can be useful as it allows such an exploratory study. It has parameters $\mu$ and $\lambda$ where this anisotropic information could be introduced, for e.g. from DWI images. For the same atrophy map, the effect of anisotropy on the brain deformation is an interesting question to explore.

Reliably simulating neurodegeneration due to $\mathrm{AD}$ and its trajectory in structural MRIs is quite challenging as we need accurate models for all three major blocks shown in Figure 1. The most difficult part is to generate accurate patterns of atrophy and its evolution with time. As we have seen from the examples in Section 5 with differential patterns of atrophy producing similar images, the atrophy estimation algorithms would benefit from a biologically motivated prior on the assumed model for regularizations. Accurate atrophy 
generation models require more information from other sources in addition to the structural MRIs. In addition to the research in biology pertaining to AD, perhaps a progress in other imaging modalities could also potentially provide information on the spread of imminent neuronal deaths. For instance $\mathrm{A} \beta$ plaques seem to occur very early at the beginning of atrophic process [37]. Studies such as brain's structural connection breakdown on AD patients using Diffusion Imaging [38][39], or functional connectivity breakdown along with the structural connectivity [40] could also provide better insight in the future. Similarly, there is ongoing research in developing good tracers to bind to tau proteins and to image in vivo the neurofibrillary tangles (NFT) [41] in AD patients. In the future we might be able to exploit such data to propose basic hypotheses of spatial atrophy distribution using multi-modal images. This could be valuable in developing suitable models for the Atrophy Generation block.

\section{Conclusions}

We developed a biophysical brain deformation model that describes the consequence of the neuronal deaths and atrophy on the brain shape changes at macroscopic scale. The model is inspired from biomechanical principles, and treats the brain parenchyma and the CSF differently to account for the fact that the CSF is produced at a very short time-scale of hours compared to the slow process of tissue atrophy taking months. We were able to achieve different deformations of the brain even with exactly the same atrophy in the brain tissue by varying the model parameters. Any desired atrophy can be prescribed at the voxel level and simulate realistic deformations of a patient specific MRI. This ability to both i) accurately prescribe complex patterns of atrophy at each voxel, and ii) to treat different tissue types differently in accordance to their biomechanical properties, was very difficult with the previous models of atrophy simulators existing in the literature. The proposed model could be used in testing hypotheses about the distribution of brain atrophy and in exploring the interaction of mechanical response of different brain tissues to neurodegener-

ation. It can also be a valuable tool to understand better the interrelationship between the underlying brain deformations corresponding to specific atrophy patterns, longitudinal MRI appearance, and the bias of various atrophy estimation methods due to the modeling error.

\section{Appendix 1: Derivation of the Conservation Law}

The atrophy rate $\tilde{a}(\mathbf{x}, t)$ at any position $\mathbf{x}$ at time $t$ for a representative elementary volume of $V_{\mathbf{x} t}$ is defined as the negative rate of change of volume per unit volume:

$$
\tilde{a}=\frac{-1}{V_{\mathbf{x} t}} \frac{\partial V_{\mathbf{x} t}}{\partial t}
$$

Let us consider a sufficiently small deformation induced in a time interval $\Delta t$. Let $\phi$ denote the deformation of the material during this time. The new position of a material particle initially at reference position $\mathbf{X}$ is given by:

$$
\mathbf{x}=\phi(\mathbf{X})=\mathbf{X}+\mathbf{u}=\mathbf{X}+\Delta t \mathbf{v}
$$


where $\mathbf{u}$ is the displacement of the particle at position $\mathbf{X}$ and $\mathbf{v}$ is the particle's velocity.

Let $V_{t}$ and $V_{t+\Delta t}$ denote the elementary volume of a material at time $t$ and $t+\Delta t$ respectively. By the definition of atrophy rate, we have,

$$
-\tilde{a} V_{t}=\frac{V_{t+\Delta t}-V_{t}}{\Delta t}
$$

Now, $a=\tilde{a} \Delta t$ which is the amount of atrophy during time $\Delta t$ is given by,

$$
\begin{aligned}
a & =\frac{V_{t}-V_{t+\Delta t}}{V_{t}} \\
& =1-\frac{V_{t+\Delta t}}{V_{t}} \\
& =1-J
\end{aligned}
$$

where $J$ is the Jacobian determinant given by,

$$
\begin{aligned}
J & =\operatorname{det}(\nabla \phi) \\
& =\operatorname{det}(\nabla(\mathbf{X}+\Delta t \mathbf{v})) \\
& =\operatorname{det}(I+\Delta t \nabla \mathbf{v})
\end{aligned}
$$

Using $\operatorname{det}(I+\epsilon A)=1+\epsilon \operatorname{tr} A+O\left(\epsilon^{2}\right)$ we can approximate $J$ as below,

$$
\begin{aligned}
J & \approx 1+\Delta t \operatorname{tr}(\nabla \mathbf{v}) \\
& =1+\Delta t \nabla \cdot \mathbf{v} \\
& =1+\nabla \cdot \mathbf{u}
\end{aligned}
$$

Now substituting $J$ in equation (7), we have:

$$
\nabla \cdot \mathbf{u}=-a
$$

\section{Acknowledgements}

1. Part of this work was funded by the European Research Council through the ERC Advanced Grant MedYMA 2011-291080.

2. Data used in the preparation of this article were obtained from the MIRIAD database. The MIRIAD investigators did not participate in analysis or writing of this report. The MIRIAD dataset is made available through the support of the UK Alzheimer's Society (Grant RF116). The original data collection was funded through an unrestricted educational grant from GlaxoSmithKline (Grant 6GKC).

3. This work benefited from the use of the Insight Segmentation and Registration Toolkit (ITK), an open source software developed as an initiative of the U.S. National Library of Medicine and available at www.itk.org. 
4. The multi-platform configuration tool CMake was used for configuring ITK and facilitating its use from our project. CMake was partially funded by the U.S. National Library of Medicine as part of the Insight Toolkit project. CMake is an open source system and it is freely available at www.cmake.org.

5. We would like to thank neurologist David Bensamoun of the Nice Resource \& Research Memory Centre (Nice, France) for providing qualitative assessment of our simulations of atrophy progression in the brain MRIs.

\section{References}

[1] H. Braak, E. Braak, Neuropathological stageing of Alzheimer-related changes, Acta Neuropathologica 82 (4) (1991) 239-259. doi:10.1007/bf00308809.

URL http://dx.doi.org/10.1007/bf00308809

[2] G. B. Frisoni, N. C. Fox, C. R. Jack, P. Scheltens, P. M. Thompson, The clinical use of structural MRI in Alzheimer disease., Nature Reviews. Neurology 6 (2) (2010) 67-77. doi:10.1038/nrneurol.2009.215. URL http://www.pubmedcentral.nih.gov/articlerender.fcgi?artid=2938772\&tool= pmcentrez\&rendertype=abstract

[3] L. S. Schneider, F. Mangialasche, N. Andreasen, H. Feldman, E. Giacobini, R. Jones, V. Mantua, P. Mecocci, L. Pani, B. Winblad, M. Kivipelto, Clinical trials and late-stage drug development for Alzheimer's disease: an appraisal from 1984 to 2014, J Intern Med 275 (3) (2014) 251-283. doi: 10.1111/joim. 12191.

URL http://dx.doi.org/10.1111/joim.12191

[4] A. D. C. Smith, W. R. Crum, D. L. Hill, N. A. Thacker, P. A. Bromiley, Biomechanical simulation of atrophy in MR images, in: Medical Imaging 2003, International Society for Optics and Photonics, 2003, pp. 481-490.

[5] O. Camara, M. Schweiger, R. I. Scahill, W. R. Crum, B. I. Sneller, J. A. Schnabel, G. R. Ridgway, D. M. Cash, D. L. G. Hill, N. C. Fox, Phenomenological model of diffuse global and regional atrophy using finite-element methods., IEEE Transactions on Medical Imaging 25 (11) (2006) 1417-30. doi: 10.1109/TMI. 2006.880588 .

URL http://www.ncbi.nlm.nih.gov/pubmed/17117771

[6] B. Karaçali, C. Davatzikos, Simulation of tissue atrophy using a topology preserving transformation model., IEEE Transactions on Medical Imaging 25 (5) (2006) 649-52. doi:10.1109/TMI . 2006 . 873221. URL http://www.ncbi.nlm.nih.gov/pubmed/16689268

[7] P. Pieperhoff, M. Südmeyer, L. Hömke, K. Zilles, A. Schnitzler, K. Amunts, Detection of structural changes of the human brain in longitudinally acquired MR images by deformation field morphometry: methodological analysis, validation and application., NeuroImage 43 (2) (2008) 269-87. doi:10.1016/ j.neuroimage.2008.07.031.

URL http://www.ncbi.nlm.nih.gov/pubmed/18706506

[8] S. Sharma, V. Noblet, F. Rousseau, F. Heitz, L. Rumbach, J. Armspach, Evaluation of brain atrophy estimation algorithms using simulated ground-truth data., Medical Image Analysis 14 (3) (2010) 37389. doi:10.1016/j.media.2010.02.002. URL http://www.ncbi.nlm.nih.gov/pubmed/20219411

[9] H. H. Damkier, P. D. Brown, J. Praetorius, Cerebrospinal Fluid Secretion by the Choroid Plexus, Physiological Reviews 93 (4) (2013) 1847-1892. doi:10.1152/physrev.00004.2013.

URL http://dx.doi.org/10.1152/physrev.00004.2013

[10] S. Sharma, F. Rousseau, F. Heitz, L. Rumbach, J. Armspach, On the estimation and correction of bias in local atrophy estimations using example atrophy simulations, Computerized Medical Imaging and Graphics 37 (7-8) (2013) 538-551. doi : http://dx.doi.org/10.1016/j.compmedimag. 2013.07.002. URL http://www.sciencedirect.com/science/article/pii/S0895611113001316 
[11] B. Khanal, M. Lorenzi, N. Ayache, X. Pennec, A Biophysical Model of Shape Changes due to Atrophy in the Brain with Alzheimer's Disease, in: P. Golland, N. Hata, C. Barillot, J. Hornegger, R. Howe (Eds.), Medical Image Computing and Computer-Assisted Intervention - MICCAI 2014, Vol. 8674 of Lecture Notes in Computer Science, Springer International Publishing, 2014, pp. 41-48. doi:10.1007/ 978-3-319-10470-6_6. URL http://dx.doi.org/10.1007/978-3-319-10470-6_6

[12] W. J. Tyler, The mechanobiology of brain function, Nature Reviews Neuroscience 13 (12) (2012) 867878. doi:10.1038/nrn3383.

URL http://dx.doi.org/10.1038/nrn3383

[13] J. Mueller, W. Tyler, Neuromechanobiology of the brain: mechanics of neuronal structure function, and pathophysiology, in: Y. Sun, D.-H. Kim, C. A. Simmons (Eds.), Integrative Mechanobiology, Cambridge University Press (CUP), 2015, pp. 347-367. doi:10.1017/cbo9781139939751.020. URL http://dx.doi.org/10.1017/cbo9781139939751.020

[14] O. Camara, J. A. Schnabel, G. R. Ridgway, W. R. Crum, A. Douiri, R. I. Scahill, D. L. Hill, N. C. Fox, Accuracy assessment of global and local atrophy measurement techniques with realistic simulated longitudinal Alzheimer's disease images, NeuroImage 42 (2) (2008) 696-709. doi: $10.1016 / j$.neuroimage .2008 .04 .259 . URL http://dx.doi.org/10.1016/j.neuroimage.2008.04.259

[15] T. Rohlfing, Transformation Model and Constraints Cause Bias in Statistics on Deformation Fields, in: Medical Image Computing and Computer-Assisted Intervention - MICCAI 2006, Springer Science Business Media, 2006, pp. 207-214. doi:10.1007/11866565_26. URL http://dx.doi.org/10.1007/11866565_26

[16] R. T. Johnson, C. J. Gibbs Jr, Creutzfeldt-Jakob disease and related transmissible spongiform encephalopathies, New England Journal of Medicine 339 (27) (1998) 1994-2004.

[17] G. K. Batchelor, An Introduction to Fluid Dynamics, Cambridge University Press (CUP), 2000. doi: 10.1017/cbo9780511800955. URL http://dx.doi.org/10.1017/cbo9780511800955

[18] I. Sack, K.-J. Streitberger, D. Krefting, F. Paul, J. Braun, The Influence of Physiological Aging and Atrophy on Brain Viscoelastic Properties in Humans, PLoS ONE 6 (9) (2011) e23451. doi:10.1371/ journal. pone. 0023451.

URL http://dx.doi.org/10.1371/journal.pone.0023451

[19] M. C. Murphy, J. Huston, C. R. Jack, K. J. Glaser, A. Manduca, J. P. Felmlee, R. L. Ehman, Decreased brain stiffness in Alzheimer's disease determined by magnetic resonance elastography, J. Magn. Reson. Imaging 34 (3) (2011) 494-498. doi:10.1002/jmri.22707. URL http://dx.doi.org/10.1002/jmri.22707

[20] M. L. Nogueira, O. Lafitte, J.-M. Steyaert, H. Bakardjian, B. Dubois, H. Hampel, L. Schwartz, Mechanical stress related to brain atrophy in Alzheimer's disease, Alzheimer's \& Dementiadoi: $10.1016 / j \cdot j a l z .2015 .03 .005$. URL http://dx.doi.org/10.1016/j.jalz.2015.03.005

[21] M. Benzi, G. H. Golub, J. Liesen, Numerical solution of saddle point problems, Acta Numerica 14 (2005) 1-137. doi:10.1017/s0962492904000212.

URL http://dx.doi.org/10.1017/s0962492904000212

[22] S. Balay, J. Brown, K. Buschelman, W. D. Gropp, D. Kaushik, M. G. Knepley, L. C. McInnes, B. F. Smith, H. Zhang, PETSc Web page, http://www.mcs.anl.gov/petsc (2013).

[23] J. Brown, M. Knepley, D. May, L. McInnes, B. Smith, Composable Linear Solvers for Multiphysics, in: 2012 11th International Symposium on Parallel and Distributed Computing (ISPDC), 2012, pp. 55-62. doi:10.1109/ISPDC.2012.16.

[24] J. E. Iglesias, C. Liu, P. M. Thompson, Z. Tu, Robust brain extraction across datasets and comparison with publicly available methods, IEEE Transactions on Medical Imaging 30 (9) (2011) 1617-1634.

[25] Y. Zhang, M. Brady, S. Smith, Segmentation of brain MR images through a hidden Markov random field model and the expectation-maximization algorithm, IEEE Transactions on Medical Imaging 20 (1) 
(2001) $45-57$.

[26] B. Patenaude, S. M. Smith, D. N. Kennedy, M. Jenkinson, A Bayesian model of shape and appearance for subcortical brain segmentation, Neuroimage 56 (3) (2011) 907-922.

[27] O. Carmichael, D. G. McLaren, D. Tommet, D. Mungas, R. N. Jones, Coevolution of brain structures in amnestic mild cognitive impairment, NeuroImage 66 (2013) 449-456. doi:10.1016/j.neuroimage. 2012.10 .029$.

URL http://www.sciencedirect.com/science/article/pii/S1053811912010324

[28] B. Fischl, D. H. Salat, E. Busa, M. Albert, M. Dieterich, C. Haselgrove, A. van der Kouwe, R. Killiany, D. Kennedy, S. Klaveness, A. Montillo, N. Makris, B. Rosen, A. M. Dale, Whole Brain Segmentation: Automated Labeling of Neuroanatomical Structures in the Human Brain, Neuron 33 (3) (2002) 341355. doi:10.1016/S0896-6273(02)00569-X.

URL http://www.sciencedirect.com/science/article/pii/S089662730200569X

[29] S. Cheng, E. C. Clarke, L. E. Bilston, Rheological properties of the tissues of the central nervous system: A review, Medical Engineering \& Physics 30 (10) (2008) 1318-1337. doi:10.1016/j .medengphy . 2008. 06.003.

URL http://dx.doi.org/10.1016/j.medengphy.2008.06.003

[30] M. Lorenzi, N. Ayache, G. Frisoni, X. Pennec, LCC-Demons: A robust and accurate symmetric diffeomorphic registration algorithm, NeuroImage 81 (2013) 470-483. doi:10.1016/j.neuroimage.2013. 04.114.

URL http://dx.doi.org/10.1016/j.neuroimage.2013.04.114

[31] F. Prados, M. J. Cardoso, K. K. Leung, D. M. Cash, M. Modat, N. C. Fox, C. A. Wheeler-Kingshott, S. Ourselin, Measuring brain atrophy with a generalized formulation of the boundary shift integral, Neurobiology of Aging 36 (2015) S81-S90. doi:10.1016/j.neurobiolaging.2014.04.035.

URL http://dx.doi.org/10.1016/j.neurobiolaging.2014.04.035

[32] NifTK software tools, accessed: 9-June-2015. URL http://cmictig.cs.ucl.ac.uk/research/software

[33] P. Freeborough, N. Fox, The boundary shift integral: an accurate and robust measure of cerebral volume changes from registered repeat MRI, IEEE Transactions on Medical Imaging 16 (5) (1997) 623-629. doi:10.1109/42.640753.

URL http://dx.doi.org/10.1109/42.640753

[34] I. B. Malone, D. Cash, G. R. Ridgway, D. G. MacManus, S. Ourselin, N. C. Fox, J. M. Schott, MIRIAD - Public release of a multiple time point Alzheimer's MR imaging dataset, NeuroImage 70 (2013) 33-36. doi:10.1016/j.neuroimage.2012.12.044.

URL http://www.sciencedirect.com/science/article/pii/S105381191201230X

[35] M. Reuter, N. J. Schmansky, H. D. Rosas, B. Fischl, Within-subject template estimation for unbiased longitudinal image analysis, NeuroImage 61 (4) (2012) 1402-1418. doi :10.1016/j .neuroimage.2012. 02.084 .

URL http://www.sciencedirect.com/science/article/pii/S1053811912002765

[36] E. Wenger, J. Mårtensson, H. Noack, N. C. Bodammer, S. Kühn, S. Schaefer, H.-J. Heinze, E. Düzel, L. Bäckman, U. Lindenberger, M. Lövdén, Comparing manual and automatic segmentation of hippocampal volumes: Reliability and validity issues in younger and older brains, Human Brain Mapping 35 (8) (2014) 4236-4248. doi:10.1002/hbm. 22473.

URL http://dx.doi.org/10.1002/hbm. 22473

[37] G. Chetelat, V. Villemagne, P. Bourgeat, K. Pike, G. Jones, D. Ames, K. Ellis, C. Szoeke, R. Martins, G. O'Keefe, O. Salvado, C. Masters, C. Rowe, Relationship between Atrophy and Beta-Amyloid Deposition in Normal Elderly Mild Cognitive Impairment and Alzheimer's Disease, Alzheimer's \& Dementia 6 (4) (2010) S45-S46. doi:10.1016/j.jalz.2010.05.129.

URL http://dx.doi.org/10.1016/j.jalz.2010.05.129

[38] G. Stebbins, C. Murphy, Diffusion tensor imaging in Alzheimer's disease and mild cognitive impairment., Behav Neurol 21 (2009) 39-49.

[39] M. Daianu, N. Jahanshad, T. M. Nir, A. W. Toga, C. R. Jack, M. W. Weiner, P. M. T. for the 
Alzheimer's Disea, Breakdown of Brain Connectivity Between Normal Aging and Alzheimer's Disease: A Structural k -Core Network Analysis, Brain Connectivity 3 (4) (2013) 407-422. doi :10.1089/brain. 2012.0137.

URL http://dx.doi.org/10.1089/brain.2012.0137

[40] M. Filippi, F. Agosta, Structural and functional network connectivity breakdown in Alzheimer's disease studied with magnetic resonance imaging techniques., J Alzheimers Dis 24 (2011) 455-74.

[41] O. G. James, P. M. Doraiswamy, S. Borges-Neto, PET Imaging of Tau Pathology in Alzheimer's Disease and Tauopathies, Front. Neurol. 6. doi:10.3389/fneur.2015.00038.

URL http://dx.doi.org/10.3389/fneur.2015.00038 